\title{
Modular, $k$-noncrossing diagrams
}

\author{
Christian M. Reidys ${ }^{a}$, Rita R. Wang ${ }^{b}$ and Albus Y. Y. Zhao ${ }^{c}$ \\ Center for Combinatorics, LPMC-TJKLC \\ Nankai University \\ Tianjin 300071 \\ P.R. China \\ ${ }^{a}$ duck@santafe.edu \\ ${ }^{b}$ wangrui@cfc.nankai.edu.cn \\ ${ }^{c}$ zyy@cfc.nankai.edu.cn
}

Submitted: Mar 27, 2010; Accepted: May 16, 2010; Published: May 20, 2010

\begin{abstract}
In this paper we compute the generating function of modular, $k$-noncrossing diagrams. A $k$-noncrossing diagram is called modular if it does not contain any isolated arcs and any arc has length at least four. Modular diagrams represent the deformation retracts of RNA tertiary structures and their properties reflect basic features of these bio-molecules. The particular case of modular noncrossing diagrams has been extensively studied. Let $\mathrm{Q}_{k}(n)$ denote the number of modular $k$-noncrossing diagrams over $n$ vertices. We derive exact enumeration results as well as the asymptotic formula $\mathrm{Q}_{k}(n) \sim c_{k} n^{-(k-1)^{2}-\frac{k-1}{2}} \gamma_{k}^{-n}$ for $k=3, \ldots, 9$ and derive a new proof of the formula $\mathrm{Q}_{2}(n) \sim 1.4848 n^{-3 / 2} 1.8489^{n}$ (Hofacker et al. 1998).
\end{abstract}

\section{Introduction}

A ribonucleic acid (RNA) molecule is the helical configuration of a primary structure of nucleotides, A, G, U and $\mathbf{C}$, together with Watson-Crick (A-U, G-C) and (U-G) base pairs. It is well-known that RNA structures exhibit cross-serial nucleotide interactions, called pseudoknots. First recognized in the turnip yellow mosaic virus in [17], they are now known to be widely conserved in functional RNA molecules.

Modular $k$-noncrossing diagrams represent a model of RNA pseudoknot structures $[5,9,11]$, that is RNA structures exhibiting cross-serial base pairings. The particular case of modular noncrossing diagrams, i.e. RNA secondary structures has been extensively studied $[7,14,21,22]$.

The main result of this paper is the computation of the generating function of modular $k$-noncrossing diagrams, $\mathbf{Q}_{k}(z)$. A diagram is a labeled graph over the vertex set $[n]=$ $\{1, \ldots, n\}$ with vertex degrees not greater than one. The standard representation of a 
diagram is derived by drawing its vertices in a horizontal line and its $\operatorname{arcs}(i, j)$ in the upper half-plane. A $k$-crossing is a set of $k$ distinct $\operatorname{arcs}\left(i_{1}, j_{1}\right),\left(i_{2}, j_{2}\right), \ldots,\left(i_{k}, j_{k}\right)$ with the property

$$
i_{1}<i_{2}<\ldots<i_{k}<j_{1}<j_{2}<\ldots<j_{k} .
$$

Similarly a $k$-nesting is a set of $k$ distinct arcs such that

$$
i_{1}<i_{2}<\ldots<i_{k}<j_{k}<\ldots j_{2}<j_{1}
$$

Let $A, B$ be two sets of arcs, then $A$ is nested in $B$ if any element of $A$ is nested in any element of $B$. A diagram without any $k$-crossings is called a $k$-noncrossing diagram. The length of an arc, $(i, j)$, is $s=j-i$, and we refer to such arc as $s$-arc. Furthermore,

- a stack of length $\sigma, S_{i, j}^{\sigma}$, is a maximal sequence of "parallel" arcs,

$$
((i, j),(i+1, j-1), \ldots,(i+(\sigma-1), j-(\sigma-1))) .
$$

$S_{i, j}^{\sigma}$ is also referred to as a $\sigma$-stack.

- a stem of size $s$ is a sequence

$$
\left(S_{i_{1}, j_{1}}^{\sigma_{1}}, S_{i_{2}, j_{2}}^{\sigma_{2}}, \ldots, S_{i_{s}, j_{s}}^{\sigma_{s}}\right)
$$

where $S_{i_{m}, j_{m}}^{\sigma_{m}}$ is nested in $S_{i_{m-1}, j_{m-1}}^{\sigma_{m-1}}$ such that any arc nested in $S_{i_{m-1}, j_{m-1}}^{\sigma_{m-1}}$ is either contained or nested in $S_{i_{m}, j_{m}}^{\sigma_{m}}$, for $2 \leqslant m \leqslant s$, see Fig. 1 .
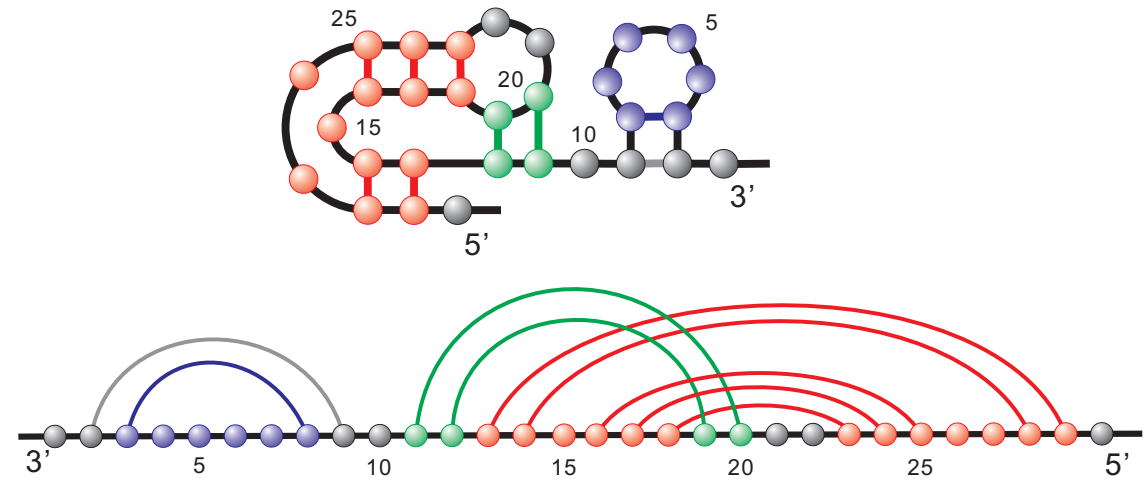

Fig. 1: Features of a modular 3-noncrossing diagram represented as planar graph (top) and in standard representation (bottom). We display a stack of length two (green), a stem of size two (red) and a 5-arc (blue).

RNA secondary structures $[8,21,22,23]$ are in the language of diagrams exactly modular, 2-noncrossing diagrams. In $[9,10,11,13]$, various classes of $k$-noncrossing diagrams have been enumerated. However the approach employed in these papers does not work for modular $k$-noncrossing diagrams. In contrast to the situation for RNA 
secondary structures, the combination of minimum arc length and nonexistence of isolated arcs poses serious difficulties. The main idea is to build modular $k$-noncrossing diagrams via inflating their colored $\mathrm{V}_{k}$-shapes, see Fig. 2 . These shapes will be discussed in detail in Section 4. The inflation gives rise to "stem-modules" over shape-arcs and is the key for the symbolic derivation of $\mathbf{Q}_{k}(z)$. One additional observation maybe worth to be pointed out: the computation of the generating function of colored shapes in Section 4, hinges on the intuition that the crossings of short arcs are relatively simple and give rise to manageable recursions. The coloring of these shapes then allows to identify the arc-configurations that require special attention during the inflation process. Our results are of importance in the context of RNA pseudoknot structures [17] and evolutionary optimization [16]. Furthermore they allow for conceptual proofs of the results in $[4,10,11,13]$.

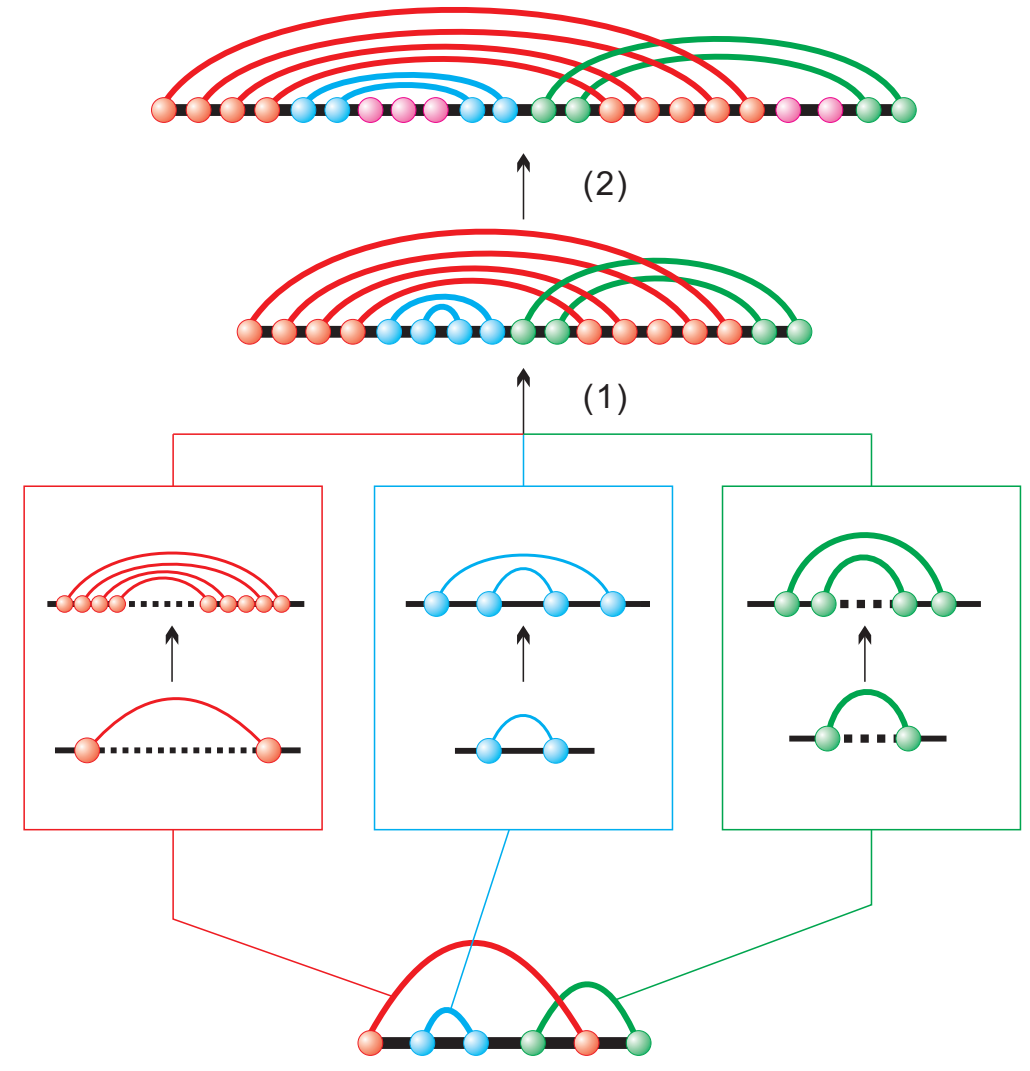

Fig. 2: Modular $k$-noncrossing diagrams: the inflation method. A modular 3-noncrossing diagram (top) is derived by inflating its $V_{3}$-shape (bottom) in two steps. First we individually inflate each shape-arc into a more complex configuration and second insert isolated vertices (purple).

The paper is organized as follows. In Section 2 we recall some basic facts on singularity analysis, the generating function of $k$-noncrossing matchings, $\mathrm{V}_{k}$-shapes and symbolic enumeration. In Section 3 we analyze modular, noncrossing diagrams and in Section 4 we compute the generating function of colored shapes. We prove our main theorem in Section 5. In Section 6 we give the proofs of Lemma 3 and Lemma 4. 


\section{Some basic facts}

\subsection{Singularity analysis}

Oftentimes, we are given a generating function without having an explicit formula of its coefficients. Singularity analysis is a framework that allows to analyze the asymptotics of these coefficients. The key to the asymptotics of the coefficients is the singularities, which raises the question on how to locate them. In the particular case of power series $f(z)=\sum_{n \geqslant 0} a_{n} z^{n}$ with nonnegative coefficients and a radius of convergence $R>0$, a theorem of Pringsheim [2, 19], guarantees a positive real dominant singularity at $z=$ $R$. As we are dealing here with combinatorial generating functions we always have this dominant singularity. We shall prove that for all our generating functions it is the unique dominant singularity. The class of theorems that deal with the deduction of information about coefficients from the generating function are called transfer-theorems [2].

Theorem 1. [2] Let $\left[z^{n}\right] f(z)$ denote the $n$-th coefficient of the power series $f(z)$ at $z=0$. (a) Suppose $f(z)=(1-z)^{-\alpha}, \alpha \in \mathbb{C} \backslash \mathbb{Z}_{\leqslant 0}$, then

$$
\begin{gathered}
{\left[z^{n}\right] f(z) \sim \frac{n^{\alpha-1}}{\Gamma(\alpha)}\left[1+\frac{\alpha(\alpha-1)}{2 n}+\frac{\alpha(\alpha-1)(\alpha-2)(3 \alpha-1)}{24 n^{2}}+\right.} \\
\left.\frac{\alpha^{2}(\alpha-1)^{2}(\alpha-2)(\alpha-3)}{48 n^{3}}+O\left(\frac{1}{n^{4}}\right)\right] .
\end{gathered}
$$

(b) Suppose $f(z)=(1-z)^{r} \log \left(\frac{1}{1-z}\right), r \in \mathbb{Z}_{\geqslant 0}$, then we have

$$
\left[z^{n}\right] f(z) \sim(-1)^{r} \frac{r !}{n(n-1) \ldots(n-r)}
$$

We use the notation

$$
(f(z)=\Theta(g(z)) \text { as } z \rightarrow \rho) \Longleftrightarrow(f(z) / g(z) \rightarrow c \text { as } z \rightarrow \rho) \text {, }
$$

where $c$ is some constant. We say a function $f(z)$ is $\Delta_{\rho}$ analytic at its dominant singularity $z=\rho$, if it analytic in some domain $\Delta_{\rho}(\phi, r)=\{z|| z|<r, z \neq r,| \operatorname{Arg}(z-\rho) \mid>\phi\}$, for some $\phi, r$, where $r>|\rho|$ and $0<\phi<\frac{\pi}{2}$. Since the Taylor coefficients have the property

$$
\forall \gamma \in \mathbb{C} \backslash 0 ; \quad\left[z^{n}\right] f(z)=\gamma^{n}\left[z^{n}\right] f\left(\frac{z}{\gamma}\right),
$$

We can, without loss of generality, reduce our analysis to the case where $z=1$ is the unique dominant singularity. The next theorem transfers the asymptotic expansion of a function near its unique dominant singularity to the asymptotic of the function's coefficients.

Theorem 2. [2] Let $f(z)$ be a $\Delta_{1}$ analytic function at its unique dominant singularity $z=1$. Let

$$
g(z)=(1-z)^{\alpha} \log ^{\beta}\left(\frac{1}{1-z}\right), \quad \alpha, \beta \in \mathbb{R} .
$$


That is we have in the intersection of a neighborhood of 1

$$
f(z)=\Theta(g(z)) \quad \text { for } z \rightarrow 1 .
$$

Then we have

$$
\left[z^{n}\right] f(z)=\Theta\left(\left[z^{n}\right] g(z)\right)
$$

\section{$2.2 \quad k$-noncrossing matchings}

A $k$-noncrossing matching is a $k$-noncrossing diagram without isolated points. Let $f_{k}(2 n)$ denote the number of $k$-noncrossing matchings. The exponential generating function of $k$-noncrossing matchings satisfies the following identity $[1,3,9]$

$$
\left.\sum_{n \geqslant 0} f_{k}(2 n) \cdot \frac{z^{2 n}}{(2 n) !}=\operatorname{det}\left[I_{i-j}(2 z)-I_{i+j}(2 z)\right]\right]_{i, j=1}^{k-1},
$$

where $I_{r}(2 z)=\sum_{j \geqslant 0} \frac{z^{2 j+r}}{j !(j+r) !}$ is the hyperbolic Bessel function of the first kind of order $r$. Eq. (2.7) combined with the fact that recursions for the coefficients of the exponential generating function translate into recursions for the coefficients of the ordinary generating function, allows us to prove:

Lemma 1. The generating function of $k$-noncrossing matchings over $2 n$ vertices, $\mathbf{F}_{k}(z)=$ $\sum_{n \geqslant 0} f_{k}(2 n) z^{n}$ is $D$-finite, [18], i.e. there exists some $e \in \mathbb{N}$ such that

$$
q_{0, k}(z) \frac{d^{e}}{d z^{e}} \mathbf{F}_{k}(z)+q_{1, k}(z) \frac{d^{e-1}}{d z^{e-1}} \mathbf{F}_{k}(z)+\cdots+q_{e, k}(z) \mathbf{F}_{k}(z)=0,
$$

where $q_{j, k}(z)$ are polynomials.

This follows from the fact that $I_{r}(2 z)$ is $D$-finite and $D$-finite power series form an algebra [18]. Lemma 1 is of importance for two reasons: first any singularity of $\mathbf{F}_{k}(z)$ is contained in the set of roots of $q_{0, k}(z)$ [18], which we denote by $R_{k}$. Second, the specific form of the ODE in eq. (2.8) is the key to derive the singular expansion of $\mathbf{F}_{k}(z)$, see Proposition 1 below.

We proceed by computing for $2 \leqslant k \leqslant 9$, the polynomials $q_{0, k}(z)$ and their roots, see Table 1 and observe that [12]

$$
f_{k}(2 n) \sim \widetilde{c}_{k} n^{-\left((k-1)^{2}+(k-1) / 2\right)}(2(k-1))^{2 n}, \quad \widetilde{c}_{k}>0, k \geqslant 2 .
$$

Equation (2.9) and Table 1 guarantee that $\mathbf{F}_{k}(z)$ has the unique dominant singularity $\rho_{k}^{2}$, where $\rho_{k}=1 /(2 k-2)$. According to Lemma $1, \mathbf{F}_{k}(z)$ is $D$-finite, whence we have analytic continuation in any simply connected domain containing zero avoiding its singularities [20]. As a result $\mathbf{F}_{k}(z)$ is $\Delta_{\rho_{k}^{2}}$ analytic as required by Theorem 2. Lemma 1 and eq. (2.9) put us in position to present the singular expansion of $\mathbf{F}_{k}(z)$ : 


\begin{tabular}{lll}
\hline$k$ & $q_{0, k}(z)$ & $R_{k}$ \\
\hline 2 & $(4 z-1) z$ & $\left\{\frac{1}{4}\right\}$ \\
3 & $(16 z-1) z^{2}$ & $\left\{\frac{1}{16}\right\}$ \\
4 & $\left(144 z^{2}-40 z+1\right) z^{3}$ & $\left\{\frac{1}{4}, \frac{1}{36}\right\}$ \\
5 & $\left(1024 z^{2}-80 z+1\right) z^{4}$ & $\left\{\frac{1}{16}, \frac{1}{64}\right\}$ \\
6 & $\left(14400 z^{3}-4144 z^{2}+140 z-1\right) z^{5}$ & $\left\{\frac{1}{4}, \frac{1}{36}, \frac{1}{100}\right\}$ \\
7 & $\left(147456 z^{3}-12544 z^{2}+224 z-1\right) z^{6}$ & $\left\{\frac{1}{16}, \frac{1}{64}, \frac{1}{144}\right\}$ \\
8 & $\left(2822400 z^{4}-826624 z^{3}+31584 z^{2}-336 z+1\right) z^{7}$ & $\left\{\frac{1}{4}, \frac{1}{36}, \frac{1}{100}, \frac{1}{196}\right\}$ \\
9 & $\left(37748736 z^{4}-3358720 z^{3}+69888 z^{2}-480 z+1\right) z^{8}$ & $\left\{\frac{1}{16}, \frac{1}{64}, \frac{1}{144}, \frac{1}{256}\right\}$ \\
\hline
\end{tabular}

Table 1: The polynomials $q_{0, k}(z)$ and their nonzero roots obtained by the MAPLE package GFUN.

Proposition 1. [6, 20] For $2 \leqslant k \leqslant 9$, the singular expansion of $\mathbf{F}_{k}(z)$ for $z \rightarrow \rho_{k}^{2}$ is given by

$$
\mathbf{F}_{k}(z)=\left\{\begin{array}{l}
P_{k}\left(z-\rho_{k}^{2}\right)+c_{k}^{\prime}\left(z-\rho_{k}^{2}\right)^{\left((k-1)^{2}+(k-1) / 2\right)-1} \log \left(z-\rho_{k}^{2}\right)(1+o(1)) \\
P_{k}\left(z-\rho_{k}^{2}\right)+c_{k}^{\prime}\left(z-\rho_{k}^{2}\right)^{\left((k-1)^{2}+(k-1) / 2\right)-1}(1+o(1)),
\end{array}\right.
$$

depending on $k$ being odd or even. Furthermore, the terms $P_{k}(z)$ are polynomials of degree not larger than $(k-1)^{2}+(k-1) / 2-1, c_{k}^{\prime}$ is some constant, and $\rho_{k}=1 /(2 k-2)$.

In our derivations the following instance of the supercritical paradigm [2] is of central importance: we are given a $D$-finite function, $f(z)$ and an algebraic function $g(u)$ satisfying $g(0)=0$. Furthermore we suppose that $f(g(u))$ has a unique real valued dominant singularity $\gamma$ and $g$ is regular in a disc with radius slightly larger than $\gamma$. Then the supercritical paradigm stipulates that the subexponential factors of $f(g(u))$ at $u=0$, given that $g(u)$ satisfies certain conditions, coincide with those of $f(z)$.

Theorem 1, Theorem 2 and Proposition 1 allow under certain conditions to obtain the asymptotics of the coefficients of supercritical compositions of the "outer" function $\mathbf{F}_{k}(z)$ and "inner" function $\psi(z)$.

Proposition 2. Let $\psi(z)$ be an algebraic, analytic function in a domain $\mathcal{D}=\{z|| z \mid \leqslant$ $r\}$ such that $\psi(0)=0$. Suppose $\gamma$ is the unique dominant singularity of $\mathbf{F}_{k}(\psi(z))$ and minimum positive real solution of $\psi(\gamma)=\rho_{k}^{2},|\gamma|<r$, where $\psi^{\prime}(\gamma) \neq 0$. Then $\mathbf{F}_{k}(\psi(z))$ has a singular expansion and

$$
\left[z^{n}\right] \mathbf{F}_{k}(\psi(z)) \sim A n^{-\left((k-1)^{2}+(k-1) / 2\right)}\left(\frac{1}{\gamma}\right)^{n},
$$

where $A$ is some constant. 


\subsection{Shapes}

Definition 1. A $\mathrm{V}_{k}$-shape is a $k$-noncrossing matching having stacks of length exactly one.

In the following we refer to $\mathrm{V}_{k}$-shape simply as shapes. That is, given a modular, $k$-noncrossing diagram, $\delta$, its shape is obtained by first replacing each stem by an arc and then removing all isolated vertices, see Fig. 3.

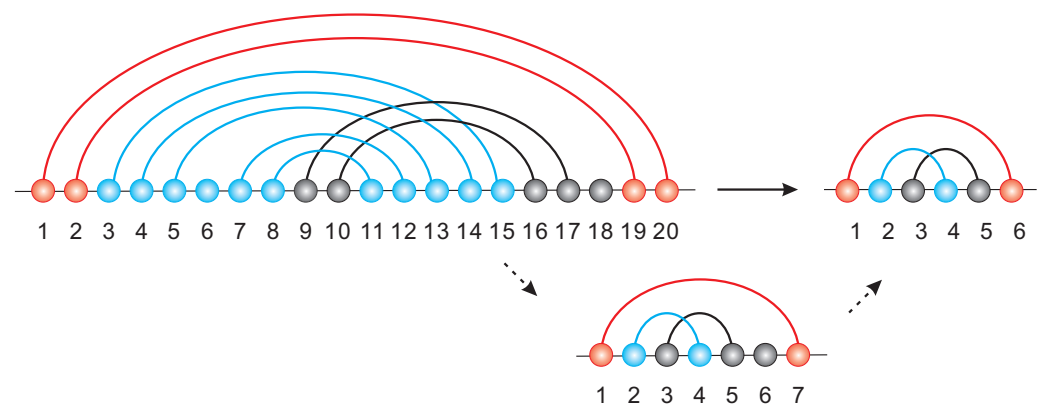

Fig. 3: From diagrams to shapes: A modular, 3-noncrossing diagram (top-left) is mapped in two steps into its $\mathrm{V}_{3}$-shape (top-right). A stem (blue) is replaced by an single shape-arc (blue).

Let $\mathcal{J}_{k}(s, m)\left(i_{k}(s, m)\right)$ denote the set (number) of the $\mathrm{V}_{k^{-}}$-shapes with $s$ arcs and $m$ 1 -arcs having the bivariate generating function

$$
\mathbf{I}_{k}(z, u)=\sum_{s \geqslant 0} \sum_{m=0}^{s} i_{k}(s, m) z^{s} u^{m} .
$$

The bivariate generating function of $i_{k}(s, m)$ and the generating function of $\mathbf{F}_{k}(z)$ are related as follows:

Lemma 2. [15] Let $k$ be natural number where $k \geqslant 2$, then the generating function $\mathbf{I}_{k}(z, u)$ satisfy

$$
\mathbf{I}_{k}(z, u)=\frac{1+z}{1+2 z-z u} \mathbf{F}_{k}\left(\frac{z(1+z)}{(1+2 z-z u)^{2}}\right)
$$

\subsection{Symbolic enumeration}

In the following we will compute the generating functions via the symbolic enumeration method [2]. For this purpose we need the notion of a combinatorial class. A combinatorial class $\left(\mathcal{C}, w_{\mathcal{C}}\right)$ is a set together with a size-function, $w_{\mathcal{C}}: \mathcal{C} \longrightarrow \mathbb{Z}^{+}$such that $\mathcal{C}_{n}=w_{\mathcal{C}}^{-1}(n)$ is finite for any $n \in \mathbb{Z}^{+}$. We write $w$ instead of $w_{e}$ and set $C_{n}=\left|\mathcal{C}_{n}\right|$. Two special combinatorial classes are $\mathcal{E}$ and $\mathcal{Z}$ which contain only one element of size 0 and 1 , respectively. The generating function of a combinatorial class $\mathcal{C}$ is given by

$$
\mathbf{C}(z)=\sum_{c \in \mathcal{C}} z^{w(c)}=\sum_{n \geqslant 0} C_{n} z^{n}
$$


where $\mathcal{C}_{n} \subset \mathcal{C}$. In particular, the generating functions of the classes $\mathcal{E}$ and $\mathcal{z}$ are $\mathbf{E}(z)=1$ and $\mathbf{Z}(z)=z$. Suppose $\mathcal{C}, \mathcal{D}$ are combinatorial classes. Then $\mathcal{C}$ is isomorphic to $\mathcal{D}$, $\mathcal{C} \cong \mathcal{D}$, if and only if $\forall n \geqslant 0,\left|\mathcal{C}_{n}\right|=\left|\mathcal{D}_{n}\right|$. In the following we shall identify isomorphic combinatorial classes and write $\mathcal{C}=\mathcal{D}$ if $\mathcal{C} \cong \mathcal{D}$. We set

- $\mathcal{C}+\mathcal{D}:=\mathcal{C} \cup \mathcal{D}$, if $\mathcal{C} \cap \mathcal{D}=\varnothing$ and for $\alpha \in \mathcal{C}+\mathcal{D}$,

$$
w_{\mathcal{C}+\mathcal{D}}(\alpha)= \begin{cases}w_{\mathcal{C}}(\alpha) & \text { if } \alpha \in \mathcal{C} \\ w_{\mathcal{D}}(\alpha) & \text { if } \alpha \in \mathcal{D} .\end{cases}
$$

- $\mathcal{C} \times \mathcal{D}:=\{\alpha=(c, d) \mid c \in \mathcal{C}, d \in \mathcal{D}\}$ and for $\alpha \in \mathcal{C} \times \mathcal{D}$,

$$
w_{\mathcal{C} \times \mathcal{D}}((c, d))=w_{\mathfrak{C}}(c)+w_{\mathcal{D}}(d) .
$$

and furthermore $\mathcal{C}^{m}:=\prod_{h=1}^{m} \mathcal{C}$ and $\operatorname{SeQ}(\mathcal{C}):=\mathcal{E}+\mathcal{C}+\mathcal{C}^{2}+\cdots$. Plainly, $\operatorname{SeQ}(\mathcal{C})$ is a combinatorial class if and only if there is no element in $\mathcal{C}$ of size 0 . We immediately observe

Proposition 3. Suppose $\mathcal{A}, \mathcal{C}$ and $\mathcal{D}$ are combinatorial classes with generating functions $\mathbf{A}(\mathbf{z}), \mathbf{C}(z)$ and $\mathbf{D}(z)$. Then

(a) $\mathcal{A}=\mathrm{C}+\mathcal{D} \Longrightarrow \mathbf{A}(z)=\mathbf{C}(z)+\mathbf{D}(z)$

(b) $\mathcal{A}=\mathrm{C} \times \mathcal{D} \Longrightarrow \mathbf{A}(z)=\mathbf{C}(z) \cdot \mathbf{D}(z)$

(c) $\mathcal{A}=\mathrm{SEQ}(\mathcal{C}) \Longrightarrow \mathbf{A}(z)=\frac{1}{1-\mathbf{C}(z)}$.

\section{Modular, noncrossing diagrams}

Let us begin by studying first the case $k=2$ [7], where the asymptotic formula

$$
\mathrm{Q}_{2}(n) \sim 1.4848 \cdot n^{-3 / 2} \cdot 1.8489^{n}
$$

has been derived. In the following we extend the result in [7] by computing the generating function explicitly. The above asymptotic formula follows then easily by means of singularity analysis.

Proposition 4. The generating function of modular, noncrossing diagrams is given by

$$
\mathbf{Q}_{2}(z)=\frac{1-z^{2}+z^{4}}{1-z-z^{2}+z^{3}+2 z^{4}+z^{6}} \cdot \mathbf{F}_{2}\left(\frac{z^{4}-z^{6}+z^{8}}{\left(1-z-z^{2}+z^{3}+2 z^{4}+z^{6}\right)^{2}}\right)
$$

and the coefficients $\mathrm{Q}_{2}(n)$ satisfy

$$
\mathrm{Q}_{2}(n) \sim c_{2} n^{-3 / 2} \gamma_{2}^{-n},
$$

where $\gamma_{2}$ is the minimal, positive real solution of $\vartheta(z)=1 / 4$, and

$$
\vartheta(z)=\frac{z^{4}-z^{6}+z^{8}}{\left(1-z-z^{2}+z^{3}+2 z^{4}+z^{6}\right)^{2}} .
$$

Here we have $\gamma_{2} \approx 1.8489$ and $c_{2} \approx 1.4848$. 
Proof. Let $Q_{2}$ denote the set of modular noncrossing diagrams, $\mathcal{J}_{2}$ the set of all $\mathrm{V}_{2}$-shapes and $\mathcal{J}_{2}(m)$ those having exactly $m$ 1-arcs. Then we have the surjective map

$$
\varphi: Q_{2} \rightarrow \mathcal{J}_{2}
$$

The map $\varphi$ is obviously surjective, inducing the partition $\mathcal{Q}_{2}=\dot{\cup}_{\gamma} \varphi^{-1}(\gamma)$, where $\varphi^{-1}(\gamma)$ is the preimage set of shape $\gamma$ under the map $\varphi$. Accordingly, we arrive at

$$
\mathrm{Q}_{2}(z)=\sum_{m \geqslant 0} \sum_{\gamma \in \mathcal{J}_{2}(m)} \mathbf{Q}_{\gamma}(z)
$$

We proceed by computing the generating function $\mathbf{Q}_{\gamma}(z)$. We shall construct $\mathbf{Q}_{\gamma}(z)$ from certain combinatorial classes as "building blocks". The latter are: $\mathcal{M}$ (stems), $\mathcal{K}$ (stacks), $\mathcal{N}$ (induced stacks), $\mathcal{L}$ (isolated vertices), $\mathcal{R}$ (arcs) and $\mathcal{Z}$ (vertices), where $\mathbf{Z}(z)=z$ and $\mathbf{R}(z)=z^{2}$. We inflate $\gamma \in \mathcal{J}_{2}(m)$ having $s$ arcs, where $s \geqslant \max \{1, m\}$, to a modular noncrossing diagram in two steps:

Claim. For any shape $\gamma \in \mathcal{J}_{2}(s, m)$ we have

$$
\begin{aligned}
\mathbf{Q}_{\gamma}(z) & =\left(\frac{\frac{z^{4}}{1-z^{2}}}{1-\frac{z^{4}}{1-z^{2}}\left(2 \frac{z}{1-z}+\left(\frac{z}{1-z}\right)^{2}\right)}\right)^{s}\left(\frac{1}{1-z}\right)^{2 s+1-m}\left(\frac{z^{3}}{1-z}\right)^{m} \\
& =(1-z)^{-1}\left(\frac{z^{4}}{\left(1-z^{2}\right)(1-z)^{2}-\left(2 z-z^{2}\right) z^{4}}\right)^{s}\left(z^{3}\right)^{m} .
\end{aligned}
$$

Step I: we inflate any shape-arc to a stack of length at least 2 and subsequently add additional stacks. The latter are called induced stacks and have to be separated by means of inserting isolated vertices, see Fig. 4. Note that during this first inflation step no
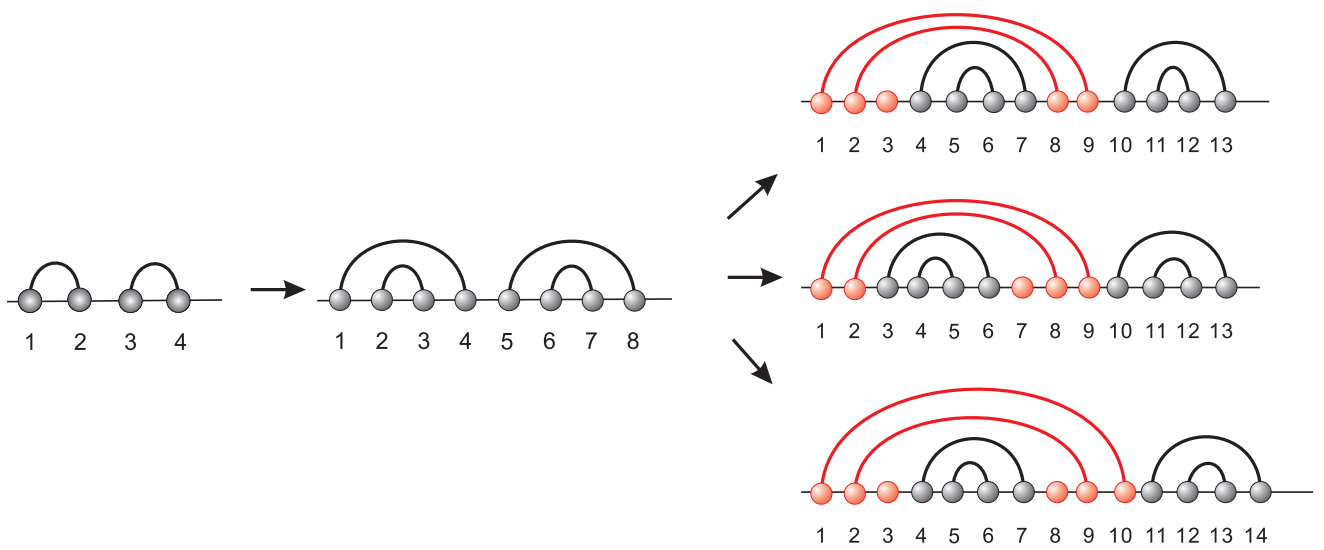

Fig. 4: Illustration of Step I.

intervals of isolated vertices, other than those necessary for separating the nested stacks are inserted. We generate 
- sequences of isolated vertices $\mathcal{L}=\operatorname{SeQ}(Z)$, where

$$
\mathbf{L}(z)=\frac{1}{1-z}
$$

- stacks, i.e.

$$
\mathcal{K}=\mathcal{R}^{2} \times \operatorname{SEQ}(\mathcal{R})
$$

with the generating function

$$
\mathbf{K}(z)=z^{4} \cdot \frac{1}{1-z^{2}}
$$

- induced stacks, i.e. stacks together with at least one nonempty interval of isolated vertices on either or both its sides.

$$
\mathcal{N}=\mathcal{K} \times\left(z \times \mathcal{L}+z \times \mathcal{L}+(Z \times \mathcal{L})^{2}\right)
$$

with generating function

$$
\mathbf{N}(z)=\frac{z^{4}}{1-z^{2}}\left(2 \frac{z}{1-z}+\left(\frac{z}{1-z}\right)^{2}\right),
$$

- stems, that is pairs consisting of a stack $\mathcal{K}$ and an arbitrarily long sequence of induced stacks

$$
\mathcal{M}=\mathcal{K} \times \operatorname{SEQ}(\mathcal{N})
$$

with generating function

$$
\mathbf{M}(z)=\frac{\mathbf{K}(z)}{1-\mathbf{N}(z)}=\frac{\frac{z^{4}}{1-z^{2}}}{1-\frac{z^{4}}{1-z^{2}}\left(2 \frac{z}{1-z}+\left(\frac{z}{1-z}\right)^{2}\right)} .
$$

Step II: we insert additional isolated vertices at the remaining $(2 s+1)$ positions. For each 1-arc at least three such isolated vertices are necessarily inserted, see Fig. 5. We

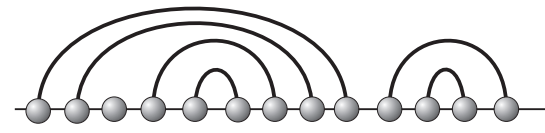

$\begin{array}{lllllllllllll}1 & 2 & 3 & 4 & 5 & 6 & 7 & 8 & 9 & 10 & 11 & 12 & 13\end{array}$

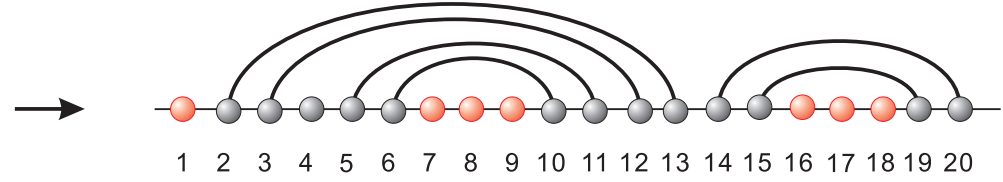

Fig. 5: Step II: the noncrossing diagram (left) obtained in (1) in Fig. 4 is inflated to a modular noncrossing diagram (right) by adding isolated vertices (red).

arrive at

$$
\mathcal{Q}_{\gamma}=(\mathcal{M})^{s} \times \mathcal{L}^{2 s+1-m} \times\left(\mathcal{Z}^{3} \times \mathcal{L}\right)^{m},
$$


where $\mathcal{Q}_{\gamma}$ is the combinatorial class of modular noncrossing diagrams having shape $\gamma \in$ $\mathrm{J}_{2}(s, m)$. Combining these generating functions the Claim follows.

Since for any $\gamma, \gamma_{1} \in \mathcal{J}_{2}(s, m)$ we have $\mathbf{Q}_{\gamma}(z)=\mathbf{Q}_{\gamma_{1}}(z)$, we derive

$$
\mathbf{Q}_{2}(z)=\sum_{m \geqslant 0} \sum_{\gamma \in J_{2}(m)} \mathbf{Q}_{\gamma}(z)=\sum_{s \geqslant 0} \sum_{m=0}^{s} i_{2}(s, m) \mathbf{Q}_{\gamma}(z) \text {. }
$$

We set

$$
\eta(z)=\frac{z^{4}}{\left(1-z^{2}\right)(1-z)^{2}-\left(2 z-z^{2}\right) z^{4}}
$$

and note that Lemma 2 guarantees

$$
\sum_{s \geqslant 0} \sum_{m=0}^{s} i_{2}(s, m) x^{s} y^{m}=\frac{1+x}{1+2 x-x y} \sum_{s \geqslant 0} f_{2}(2 s)\left(\frac{x(1+x)}{(1+2 x-x y)^{2}}\right)^{s} .
$$

Therefore, setting $x=\eta(z)$ and $y=z^{3}$ we arrive at

$$
\mathbf{Q}_{2}(z)=\frac{1-z^{2}+z^{4}}{1-z-z^{2}+z^{3}+2 z^{4}+z^{6}} \cdot \mathbf{F}_{2}\left(\frac{z^{4}-z^{6}+z^{8}}{\left(1-z-z^{2}+z^{3}+2 z^{4}+z^{6}\right)^{2}}\right)
$$

By Lemma 1, $\mathbf{Q}_{2}(z)$ is D-finite. Pringsheim's Theorem [19] guarantees that $\mathbf{Q}_{2}(z)$ has a dominant real positive singularity $\gamma_{2}$. We verify that $\gamma_{2}$ which is the unique solution of minimum modulus of the equation $\vartheta(z)=\rho_{2}^{2}$, where $\rho_{2}^{2}$ is the unique dominant singularity of $\mathbf{F}_{2}(z)$ and $\rho_{2}=1 / 2$. Furthermore we observe that $\gamma_{2}$ is the unique dominant singularity of $\mathbf{Q}_{2}(z)$. It is straightforward to verify that $\vartheta^{\prime}\left(\gamma_{2}\right) \neq 0$. According to Proposition 2, we therefore have

$$
\mathrm{Q}_{2}(n) \sim c_{2} n^{-3 / 2} \gamma_{2}^{-n}
$$

and the proof of Proposition 4 is complete.

\section{Colored shapes}

In the following we shall assume that $k>2$, unless stated otherwise. The key to compute the generating function of modular $k$-noncrossing diagrams are certain refinements of their $\mathrm{V}_{k}$-shapes. These refined shapes are called colored shapes and obtained by distinguishing a variety of crossings of 2-arcs, i.e. arcs of the form $(i, i+2)$. Each such class requires its specific inflation-procedure in Theorem 3.

Let us next have a closer look at these combinatorial classes (colors):

- $\mathbf{C}_{1}$ the class of of 1-arcs,

- $\mathbf{C}_{2}$ the class of arc-pairs consisting of mutually crossing 2-arcs,

- $\mathbf{C}_{3}$ the class of arc-pairs $(\alpha, \beta)$ where $\alpha$ is the unique 2 -arc crossing $\beta$ and $\beta$ has length at least three.

- $\mathbf{C}_{4}$ the class of arc-triples $\left(\alpha_{1}, \beta, \alpha_{2}\right)$, where $\alpha_{1}$ and $\alpha_{2}$ are 2-arcs that cross $\beta$. 

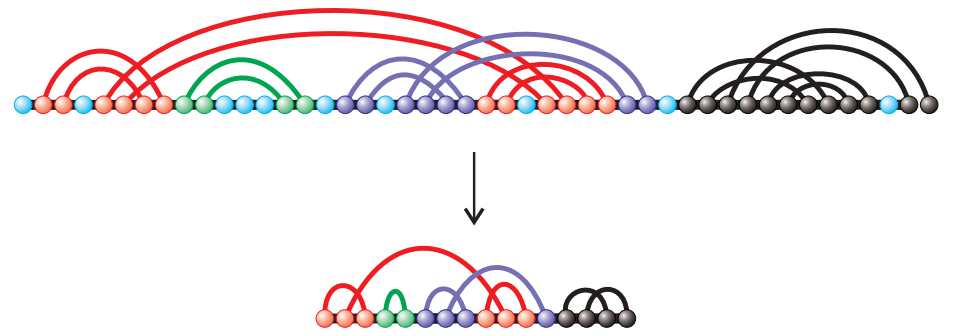

Fig. 6: Colored $\mathrm{V}_{k}$-shapes: a modular 3-noncrossing diagram (top) and its colored $\mathrm{V}_{3}$-shape (bottom). In the resulting $\mathrm{V}_{3}$-shape we color the four classes as follows: $\mathbf{C}_{1}$ (green), $\mathbf{C}_{2}$ (black), $\mathbf{C}_{3}$ (blue) and $\mathbf{C}_{4}$ (red).

In Fig. 6 we illustrate how these classes are induced by modular $k$-noncrossing diagrams.

Let us refine $\mathrm{V}_{k}$-shapes in two stages. For this purpose let $\mathcal{J}_{k}\left(s, u_{1}, u_{2}\right)$ and $i_{k}\left(s, u_{1}, u_{2}\right)$ denote the set and cardinality of $\mathrm{V}_{k}$-shapes having $s$ arcs, $u_{1} 1$-arcs and $u_{2}$ pairs of mutually crossing 2-arcs. Our first objective consists in computing the generating function

$$
\mathbf{W}_{k}(x, y, w)=\sum_{s \geqslant 0} \sum_{u_{1}=0}^{s} \sum_{u_{2}=0}^{\left\lfloor\frac{s-u_{1}}{2}\right\rfloor} i_{k}\left(s, u_{1}, u_{2}\right) x^{s} y^{u_{1}} w^{u_{2}}
$$

That is, we first take the classes $\mathbf{C}_{1}$ and $\mathbf{C}_{2}$ into account.

Lemma 3. For $k>2$, the coefficients $i_{k}\left(s, u_{1}, u_{2}\right)$ satisfy

$$
\begin{aligned}
i_{k}\left(s, u_{1}, u_{2}\right) & =0 \quad \text { for } u_{1}+2 u_{2}>s \\
\sum_{u_{2}=0}^{\left\lfloor\frac{s-u_{1}}{2}\right\rfloor} i_{k}\left(s, u_{1}, u_{2}\right) & =i_{k}\left(s, u_{1}\right),
\end{aligned}
$$

where $i_{k}\left(s, u_{1}\right)$ denotes the number of $\mathrm{V}_{k}$-shapes having s arcs, $u_{1}$ 1-arcs. Furthermore we have the recursion:

$$
\begin{aligned}
\left(u_{2}+1\right) i_{k}\left(s+1, u_{1}, u_{2}+1\right)= & \left(u_{1}+1\right) i_{k}\left(s, u_{1}+1, u_{2}\right) \\
& +\left(u_{1}+1\right) i_{k}\left(s-1, u_{1}+1, u_{2}\right) .
\end{aligned}
$$

The solution of eq. (4.1)-(4.3) is unique.

The proof of Lemma 3 is given in Section 6. We next compute $\mathbf{W}_{k}(x, y, w)$.

Proposition 5. For $k>2$, we have

$$
\mathbf{W}_{k}(x, y, w)=(1+x) v \mathbf{F}_{k}\left(x(1+x) v^{2}\right),
$$

where $v=\left((1-w) x^{3}+(1-w) x^{2}+(2-y) x+1\right)^{-1}$. 
Proof. According to Lemma 2, we have

$$
\mathbf{I}_{k}(z, u)=\frac{1+z}{1+2 z-z u} \mathbf{F}_{k}\left(\frac{z(1+z)}{(1+2 z-z u)^{2}}\right) .
$$

This generating function is connected to $\mathbf{W}_{k}(x, y, z)$ via eq. (4.2) of Lemma 3 as follows: setting $w=1$, we have $\mathbf{W}_{k}(x, y, 1)=\mathbf{I}_{k}(x, y)$. The recursion of eq. (4.3) gives rise to the partial differential equation

$$
\frac{\partial \mathbf{W}_{k}(x, y, w)}{\partial w}=x \frac{\partial \mathbf{W}_{k}(x, y, w)}{\partial y}+x^{2} \frac{\partial \mathbf{W}_{k}(x, y, w)}{\partial y} .
$$

We next show

- the function

$$
\begin{aligned}
\mathbf{W}_{k}^{*}(x, y, w)= & \frac{(1+x)}{(1-w) x^{3}+(1-w) x^{2}+(2-y) x+1} \times \\
& \mathbf{F}_{k}\left(\frac{(1+x) x}{\left((1-w) x^{3}+(1-w) x^{2}+(2-y) x+1\right)^{2}}\right)
\end{aligned}
$$

is a solution of eq. (4.5),

- its coefficients, $i_{k}^{*}\left(s, u_{1}, u_{2}\right)=\left[x^{s} y^{u_{1}} w^{u_{2}}\right] \mathbf{W}_{k}^{*}(x, y, w)$, satisfy

$$
i_{k}^{*}\left(s, u_{1}, u_{2}\right)=0 \quad \text { for } \quad u_{1}+2 u_{2}>s,
$$

- $\mathbf{W}_{k}^{*}(x, y, 1)=\mathbf{I}_{k}(x, y)$.

Firstly,

$$
\begin{aligned}
& \frac{\partial \mathbf{W}_{k}^{*}(x, y, w)}{\partial y}=u \mathbf{F}_{k}(u)+2 u \mathbf{F}_{k}^{\prime}(u) \\
& \frac{\partial \mathbf{W}_{k}^{*}(x, y, w)}{\partial w}=x(1+x) u \mathbf{F}_{k}(u)+2 x(1+x) u \mathbf{F}_{k}^{\prime}(u)
\end{aligned}
$$

where

$$
u=\frac{x(1+x)}{\left((1-w) x^{3}+(1-w) x^{2}+(2-y) x+1\right)^{2}}
$$

and $\mathbf{F}_{k}^{\prime}(u)=\sum_{n \geqslant 0} n f_{k}(2 n)(u)^{n}$. Consequently, we derive

$$
\frac{\partial \mathbf{W}_{k}^{*}(x, y, w)}{\partial w}=x \frac{\partial \mathbf{W}_{k}^{*}(x, y, w)}{\partial y}+x^{2} \frac{\partial \mathbf{W}_{k}^{*}(x, y, w)}{\partial y} .
$$

Secondly we prove $i_{k}^{*}\left(s, u_{1}, u_{2}\right)=0$ for $u_{1}+2 u_{2}>s$. To this end we observe that $\mathbf{W}_{k}^{*}(x, y, w)$ is a power series, since it is analytic in $(0,0,0)$. It now suffices to note that the indeterminants $y$ and $w$ only appear in form of products $x y$ and $x^{2} w$ or $x^{3} w$. Thirdly, the equality $\mathbf{W}_{k}^{*}(x, y, 1)=\mathbf{I}_{k}(x, y)$ is obvious.

Claim.

$$
\mathbf{W}_{k}^{*}(x, y, w)=\mathbf{W}_{k}(x, y, w)
$$


By construction the coefficients $i_{k}^{*}\left(s, u_{1}, u_{2}\right)$ satisfy eq. (4.3) and we just proved that $i_{k}^{*}\left(s, u_{1}, u_{2}\right)=0$ for $u_{1}+2 u_{2}>s$. In view of $\mathbf{W}_{k}^{*}(x, y, 1)=\mathbf{I}_{k}(x, y)$ we have

$$
\forall s, u_{1} ; \quad \sum_{u_{2}=0}^{\left\lfloor\frac{s-u_{1}}{2}\right\rfloor} i_{k}^{*}\left(s, u_{1}, u_{2}\right)=i_{k}\left(s, u_{1}\right) .
$$

Using these three properties, Lemma 3 implies

$$
\forall s, u_{1}, u_{2} \geqslant 0 ; \quad i_{k}^{*}\left(s, u_{1}, u_{2}\right)=i_{k}\left(s, u_{1}, u_{2}\right),
$$

whence the Claim and the proposition is proved.

In addition to $\mathbf{C}_{1}$ and $\mathbf{C}_{2}$, we consider next the classes $\mathbf{C}_{3}$ and $\mathbf{C}_{4}$. For this purpose we have to identify two new recursions, see Lemma 4 . Setting $\vec{u}=\left(u_{1}, \ldots, u_{4}\right)$ we denote by $\mathcal{J}_{k}(s, \vec{u})$ and $i_{k}(s, \vec{u})$, the set and number of colored $\mathrm{V}_{k^{-}}$-shapes over $s$ arcs, containing $u_{i}$ elements of class $\mathbf{C}_{i}$, where $1 \leqslant i \leqslant 4$. The key result is

Lemma 4. For $k>2$, the coefficients $i_{k}(s, \vec{u})$ satisfy

$$
\begin{aligned}
i_{k}\left(s, u_{1}, u_{2}, u_{3}, u_{4}\right) & =0 \text { for } u_{1}+2 u_{2}+2 u_{3}+3 u_{4}>s \\
\sum_{u_{3}, u_{4} \geqslant 0} i_{k}\left(s, u_{1}, u_{2}, u_{3}, u_{4}\right) & =i_{k}\left(s, u_{1}, u_{2}\right) .
\end{aligned}
$$

Furthermore we have the recursions

$$
\begin{aligned}
&\left(u_{3}+1\right) i_{k}\left(s+1, u_{1}, u_{2}, u_{3}+1, u_{4}\right)= \\
& 2 u_{1} i_{k}\left(s-1, u_{1}, u_{2}, u_{3}, u_{4}\right) \\
&+ 4\left(u_{2}+1\right) i_{k}\left(s-1, u_{1}, u_{2}+1, u_{3}, u_{4}\right) \\
&+ 4\left(u_{2}+1\right) i_{k}\left(s-1, u_{1}, u_{2}+1, u_{3}-1, u_{4}\right) \\
&+ 4\left(u_{2}+1\right) i_{k}\left(s-2, u_{1}, u_{2}+1, u_{3}-1, u_{4}\right) \\
&+ 2\left(u_{3}+1\right) i_{k}\left(s, u_{1}, u_{2}, u_{3}+1, u_{4}\right) \\
&+ 2 u_{3} i_{k}\left(s-1, u_{1}, u_{2}, u_{3}, u_{4}\right) \\
&+ 6\left(u_{3}+1\right) i_{k}\left(s-1, u_{1}, u_{2}, u_{3}+1, u_{4}\right) \\
&+ 2\left(u_{3}+1\right) i_{k}\left(s-2, u_{1}, u_{2}, u_{3}+1, u_{4}\right) \\
&+ 2 u_{3} i_{k}\left(s-2, u_{1}, u_{2}, u_{3}, u_{4}\right) \\
&+ 4\left(u_{4}+1\right) i_{k}\left(s, u_{1}, u_{2}, u_{3}-1, u_{4}+1\right) \\
&+ 4\left(u_{4}+1\right) i_{k}\left(s-1, u_{1}, u_{2}, u_{3}-1, u_{4}+1\right) \\
&+ 4 u_{4} i_{k}\left(s-1, u_{1}, u_{2}, u_{3}, u_{4}\right) \\
&+ 4\left(u_{4}+1\right) i_{k}\left(s-1, u_{1}, u_{2}, u_{3}, u_{4}+1\right) \\
&+ 4 u_{4} i_{k}\left(s-2, u_{1}, u_{2}, u_{3}, u_{4}\right) \\
&+ 2\left(u_{4}+1\right) i_{k}\left(s-2, u_{1}, u_{2}, u_{3}, u_{4}+1\right) \\
&+\left(2 s-2 u_{1}-4 u_{2}-4 u_{3}-6 u_{4}\right) i_{k}\left(s, u_{1}, u_{2}, u_{3}, u_{4}\right) \\
&+ 2\left(2(s-1)-2 u_{1}-4 u_{2}-4 u_{3}-6 u_{4}\right) i_{k}\left(s-1, u_{1}, u_{2}, u_{3}, u_{4}\right) \\
&+\left(2(s-2)-4 u_{2}-4 u_{3}-6 u_{4}\right) i_{k}\left(s-2, u_{1}, u_{2}, u_{3}, u_{4}\right) \\
&
\end{aligned}
$$


and

$$
\begin{aligned}
2\left(u_{4}+1\right) i_{k}\left(s+1, u_{1}, u_{2}, u_{3}, u_{4}+1\right)= & \left(u_{3}+1\right) i_{k}\left(s, u_{1}, u_{2}, u_{3}+1, u_{4}\right) \\
& +2\left(u_{2}+1\right)_{k}\left(s, u_{1}, u_{2}+1, u_{3}, u_{4}\right) .
\end{aligned}
$$

The sequence satisfying eq. (4.11)-(4.14) is unique.

The proof of Lemma 4 is given in Section 6. It is obtained by removing a specific arc in a labeled $\mathbf{C}_{3}$-element or a labeled $\mathbf{C}_{4}$-element and accounting of the resulting arcconfigurations.

Proposition 5 and Lemma 4 put us in position to compute the generating function of colored $\mathrm{V}_{k}$-shapes

$$
\mathbf{I}_{k}(x, y, z, w, t)=\sum_{s, u_{1}, u_{2}, u_{3}, u_{4}} i_{k}(s, \vec{u}) x^{s} y^{u_{1}} z^{u_{2}} w^{u_{3}} t^{u_{4}}
$$

Proposition 6. For $k>2$, the generating function of colored $\mathrm{V}_{k}$-shapes is given by

$$
\mathbf{I}_{k}(x, y, z, w, t)=\frac{1+x}{\theta} \mathbf{F}_{k}\left(\frac{x\left(1+(2 w-1) x+(t-1) x^{2}\right)}{\theta^{2}}\right),
$$

where $\theta=1-(y-2) x+(2 w-z-1) x^{2}+(2 w-z-1) x^{3}$.

Proof. The first recursion of Lemma 4 implies the partial differential equation

$$
\begin{aligned}
\frac{\partial \mathbf{I}_{k}}{\partial w} & =\frac{\partial \mathbf{I}_{k}}{\partial x}\left(2 x^{2}+4 x^{3}+2 x^{4}\right)-\frac{\partial \mathbf{I}_{k}}{\partial y}\left(2 x y+2 x^{2} y\right) \\
& +\frac{\partial \mathbf{I}_{k}}{\partial z}\left(-4 x z+4 x^{2} w+4 x^{2}-4 x^{3} z-8 x^{2} z+4 x^{3} w\right) \\
& +\frac{\partial \mathbf{I}_{k}}{\partial w}\left(-4 x w+2 x-6 x^{2} w+6 x^{2}-2 x^{3} w+2 x^{3}\right) \\
& +\frac{\partial \mathbf{I}_{k}}{\partial t}\left(-6 x t+4 x w-8 x^{2} t+4 x^{2} w+4 x^{2}-2 x^{3} t+2 x^{3}\right) .
\end{aligned}
$$

Analogously, the second recursion of Lemma 4 gives rise to the partial differential equation

$$
2 \frac{\partial \mathbf{I}_{k}}{\partial t}=\frac{\partial \mathbf{I}_{k}}{\partial w} x+\frac{\partial \mathbf{I}_{k}}{\partial z} 2 x .
$$

Aside from being a solution of eq. (4.17) and eq. (4.18), we take note of the fact that eq. (4.12) of Lemma 4 is equivalent to

$$
\mathbf{I}_{k}(x, y, z, 1,1)=\mathbf{W}_{k}(x, y, z) .
$$

We next show 
- The function

$$
\begin{aligned}
\mathbf{I}_{k}^{*}(x, y, z, w, t) & =\frac{1+x}{1-(y-2) x+(2 w-z-1) x^{2}+(2 w-z-1) x^{3}} \times \\
& \mathbf{F}_{k}\left(\frac{x\left(1+(2 w-1) x+(t-1) x^{2}\right)}{\left(1-(y-2) x+(2 w-z-1) x^{2}+(2 w-z-1) x^{3}\right)^{2}}\right)
\end{aligned}
$$

is a solution of eq. (4.17) and eq. (4.18),

- its coefficients, $i_{k}^{*}\left(s, u_{1}, u_{2}, u_{3}, u_{4}\right)=\left[x^{s} y^{u_{1}} z^{u_{2}} w^{u_{3}} t^{u_{4}}\right] \mathbf{I}_{k}^{*}(x, y, z, w, t)$, satisfy

$$
i_{k}^{*}\left(s, u_{1}, u_{2}, u_{3}, u_{4}\right)=0 \quad \text { for } \quad u_{1}+2 u_{2}+2 u_{3}+3 u_{4}>s
$$

- $\mathbf{I}_{k}^{*}(x, y, z, 1,1)=\mathbf{W}_{k}(x, y, z)$.

We verify by direct computation that $\mathbf{I}_{k}^{*}(x, y, z, w, t)$ satisfies eq. (4.17) as well as eq. (4.18). Next we prove $i_{k}^{*}\left(s, u_{1}, u_{2}, u_{3}, u_{4}\right)=0$ for $u_{1}+2 u_{2}+2 u_{3}+3 u_{4}>s$. Since $\mathbf{I}_{k}^{*}(x, y, z, w, t)$ is analytic in $(0,0,0,0,0)$, it is a power series. As the indeterminants $y, z, w$ and $t$ appear only in form of products $x y, x^{2} z$ or $x^{3} z, x^{2} w$ or $x^{3} w$, and $x^{3} t$, respectively, the assertion follows.

Claim.

$$
\mathbf{I}_{k}^{*}(x, y, z, w, t)=\mathbf{I}_{k}(x, y, z, w, t) .
$$

By construction, $i_{k}^{*}(s, \vec{u})$ satisfies the recursions eq. (4.13) and eq. (4.14) as well as $i_{k}^{*}\left(s, u_{1}, u_{2}, u_{3}, u_{4}\right)=0$ for $u_{1}+2 u_{2}+2 u_{3}+3 u_{4}>s$. Eq. (4.19) implies

$$
\sum_{u_{3}, u_{4} \geqslant 0} i_{k}^{*}\left(s, u_{1}, u_{2}, u_{3}, u_{4}\right)=i_{k}\left(s, u_{1}, u_{2}\right)
$$

Using these properties we can show via Lemma 4,

$$
\forall s, u_{1}, u_{2}, u_{3}, u_{4} \geqslant 0 ; \quad i_{k}^{*}\left(s, u_{1}, u_{2}, u_{3}, u_{4}\right)=i_{k}\left(s, u_{1}, u_{2}, u_{3}, u_{4}\right)
$$

and the proposition is proved.

\section{The main theorem}

We are now in position to compute $\mathbf{Q}_{k}(z)$. All technicalities aside, we already introduced the main the strategy in the proof of Proposition 4: as in the case $k=2$ we shall take care of all "critical" arcs by specific inflations.

Theorem 3. Suppose $k>2$, then

$$
\mathbf{Q}_{k}(z)=\frac{1-z^{2}+z^{4}}{q(z)} \mathbf{F}_{k}(\vartheta(z)),
$$


where

$$
\begin{aligned}
& q(z)=1-z-z^{2}+z^{3}+2 z^{4}+z^{6}-z^{8}+z^{10}-z^{12} \\
& \vartheta(z)=\frac{z^{4}\left(1-z^{2}-z^{4}+2 z^{6}-z^{8}\right)}{q(z)^{2}} .
\end{aligned}
$$

Furthermore, for $3 \leqslant k \leqslant 9, \mathrm{Q}_{k}(n)$ satisfies

$$
\mathrm{Q}_{k}(n) \sim c_{k} n^{-\left((k-1)^{2}+(k-1) / 2\right)} \gamma_{k}^{-n}, \quad \text { for some } c_{k}>0,
$$

where $\gamma_{k}$ is the minimal, positive real solution of $\vartheta(z)=\rho_{k}^{2}$, see Table 2 .

\begin{tabular}{llllllll}
\hline$k$ & 3 & 4 & 5 & 6 & 7 & 8 & 9 \\
\hline$\theta(n)$ & $n^{-5}$ & $n^{-\frac{21}{2}}$ & $n^{-18}$ & $n^{-\frac{55}{2}}$ & $n^{-39}$ & $n^{-\frac{105}{2}}$ & $n^{-68}$ \\
\hline$\gamma_{k}^{-1}$ & 2.5410 & 3.0132 & 3.3974 & 3.7319 & 4.0327 & 4.3087 & 4.5654 \\
\hline
\end{tabular}

Table 2: Exponential growth rates $\gamma_{k}^{-1}$ and subexponential factors $\theta(n)$, for modular, $k$ noncrossing diagrams.

Proof. Let $\mathcal{Q}_{k}$ denote the set of modular, $k$-noncrossing diagrams and let $\mathcal{J}_{k}$ and $\mathcal{J}_{k}(s, \vec{u})$ denote the set of all $\mathrm{V}_{k}$-shapes and those having $s$ arcs and $u_{i}$ elements belonging to class $\mathbf{C}_{i}$, where $1 \leqslant i \leqslant 4$. Then we have the surjective map,

$$
\varphi_{k}: \mathcal{Q}_{k} \rightarrow \mathcal{J}_{k}
$$

inducing the partition $Q_{k}=\dot{U}_{\gamma} \varphi_{k}^{-1}(\gamma)$, where $\varphi_{k}^{-1}(\gamma)$ is the preimage set of shape $\gamma$ under the map $\varphi_{k}$. This partition allows us to organize $\mathbf{Q}_{k}(z)$ with respect to colored $\mathrm{V}_{k}$-shapes, $\gamma$, as follows:

$$
\mathrm{Q}_{k}(z)=\sum_{s, \vec{u}} \sum_{\gamma \in \mathcal{J}_{k}(s, \vec{u})} \mathbf{Q}_{\gamma}(z)
$$

We proceed by computing the generating function $\mathbf{Q}_{\gamma}(z)$ following the strategy of Proposition 4, also using the notation therein. The key point is that the inflation-procedures are specific to the $\mathbf{C}_{i}$-classes. We next inflate all "critical" arcs, i.e. arcs that require the insertion of additional isolated vertices in order to satisfy the minimum arc length condition.

Claim 1. For a shape $\gamma \in \mathcal{J}_{k}(s, \vec{u})$ we have

$$
\begin{aligned}
\mathbf{Q}_{\gamma}(z) & =\mathbf{C}_{1}(z)^{u_{1}} \cdot \mathbf{C}_{2}(z)^{u_{2}} \cdot \mathbf{C}_{3}(z)^{u_{3}} \cdot \mathbf{C}_{4}(z)^{u_{4}} \cdot \mathbf{S}(z) \\
& =\frac{1}{1-z} \varsigma_{0}(z)^{s} \varsigma_{1}(z)^{u_{1}} \varsigma_{2}(z)^{u_{2}} \varsigma_{3}(z)^{u_{3}} \varsigma_{4}(z)^{u_{4}}
\end{aligned}
$$


where

$$
\begin{aligned}
& \varsigma_{0}(z)=\frac{z^{4}}{1-2 z+2 z^{3}-z^{4}-2 z^{5}+z^{6}}, \quad \varsigma_{1}(z)=z^{3} \\
& \varsigma_{2}(z)=\frac{z\left(1-4 z^{3}+2 z^{4}+8 z^{5}-6 z^{6}-7 z^{7}+8 z^{8}+2 z^{9}-4 z^{10}+z^{11}\right)}{1-z} \\
& \varsigma_{3}(z)=z\left(2-2 z^{2}+z^{3}+2 z^{4}-z^{5}\right) \\
& \varsigma_{4}(z)=z^{2}\left(5-4 z-3 z^{2}+6 z^{3}+2 z^{4}-4 z^{5}+z^{6}\right) .
\end{aligned}
$$

We show how to inflate a shape into a modular $k$-noncrossing diagram, distinguishing specific classes of shape-arcs. For this purpose we refer to a stem different from a 2-stack as a $\dagger$-stem. Accordingly, the combinatorial class of $\dagger$-stems is given by $\left(\mathcal{M}-\mathcal{R}^{2}\right)$.

- $\mathbf{C}_{1}$-class: here we insert isolated vertices, see Fig. 7, and obtain immediately

$$
\mathbf{C}_{1}(z)=\frac{z^{3}}{1-z}
$$

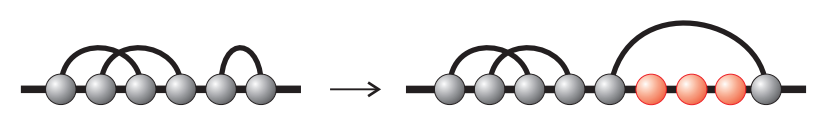

Fig. 7: $\mathbf{C}_{1}$-class: insertion of at least three vertices (red)

- $\mathbf{C}_{2}$-class: any such element is a pair $((i, i+2),(i+1, i+3))$ and we shall distinguish the following scenarios:

- both arcs are inflated to stacks of length two, see Fig. 8. Ruling out the cases where no isolated vertex is inserted and the two scenarios, where there is no insertion into the interval $[i+1, i+2]$ and only in either $[i, i+1]$ or $[i+2, i+3]$, see Fig. 8, we arrive at

$$
\mathcal{C}_{2}^{(\mathrm{a})}=\mathcal{R}^{4} \times\left[(\operatorname{SEQ}(\boldsymbol{Z}))^{3}-\mathcal{E}-2(\boldsymbol{Z} \times \operatorname{SEQ}(\boldsymbol{Z}))\right]
$$

This combinatorial class has the generating function

$$
\mathrm{C}_{2}^{(\mathrm{a})}(z)=z^{8}\left(\left(\frac{1}{1-z}\right)^{3}-1-\frac{2 z}{1-z}\right) .
$$

- one arc, $(i+1, i+3)$ or $(i, i+2)$ is inflated to a 2 -stack, while its counterpart is inflated to an arbitrary $\dagger$-stem, see Fig. 9. Ruling out the cases where no vertex is inserted in $[i+1, i+2]$ and $[i+2, i+3]$ or $[i, i+1]$ and $[i+2, i+3]$, we obtain

$$
\mathcal{C}_{2}^{(\mathrm{b})}=2 \cdot\left[\mathcal{R}^{2} \times\left(\mathcal{M}-\mathcal{R}^{2}\right) \times\left((\operatorname{SEQ}(Z))^{2}-\mathcal{E}\right) \times \operatorname{SEQ}(Z)\right]
$$




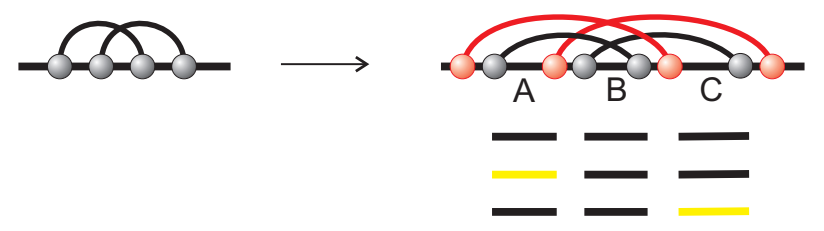

Fig. 8: $\mathbf{C}_{2}$-class: inflation of both arcs to 2-stacks. Inflated arcs are colored red while the original arcs of the shape are colored black. We set $A=[i+1, i+2], B=[i+2, i+3]$ and $C=[i+2, i+3]$ and illustrate the "bad" insertion scenarios as follows: an insertion of some isolated vertices is represented by a yellow segment and no insertion by a black segment. See the text for details.

having the generating function

$$
\mathrm{C}_{2}^{(\mathrm{b})}(z)=2 z^{4}\left(\frac{\frac{z^{4}}{1-z^{2}}}{1-\frac{z^{4}}{1-z^{2}}\left(\frac{2 z}{1-z}+\left(\frac{z}{1-z}\right)^{2}\right)}-z^{4}\right)\left(\left(\frac{1}{1-z}\right)^{2}-1\right) \frac{1}{1-z} .
$$

Fig. 9: $\mathbf{C}_{2}$-class: inflation of only one arc to a 2-stack. Arc-coloring and labels as in Fig. 8

- both arcs are inflated to an arbitrary †-stem, respectively, see Fig. 10. In this case the insertion of isolated vertices is arbitrary, whence

$$
\mathcal{C}_{2}^{(\mathrm{c})}=\left(\mathcal{M}-\mathcal{R}^{2}\right)^{2} \times(\operatorname{SEQ}(Z))^{3},
$$

with generating function

$$
\mathbf{C}_{2}^{(\mathrm{c})}(z)=\left(\frac{\frac{z^{4}}{1-z^{2}}}{1-\frac{z^{4}}{1-z^{2}}\left(\frac{2 z}{1-z}+\left(\frac{z}{1-z}\right)^{2}\right)}-z^{4}\right)^{2}\left(\frac{1}{1-z}\right)^{3} .
$$

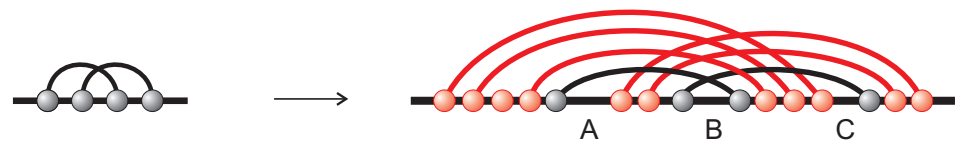

Fig. 10: $\mathbf{C}_{2}$-class: inflation of both arcs to an arbitrary $\dagger$-stem. Arc-coloring and labels as in Fig. 8

As the above scenarios are mutually exclusive, the generating function of the $\mathrm{C}_{2}$-class is given by

$$
\mathbf{C}_{2}(z)=\mathbf{C}_{2}^{(\mathrm{a})}(z)+\mathbf{C}_{2}^{(\mathrm{b})}(z)+\mathbf{C}_{2}^{(\mathrm{c})}(z) .
$$

Furthermore note that both arcs of an $\mathcal{C}_{2}$-element are inflated in the cases (a), (b) and (c). 
- $\mathbf{C}_{3}$-class: this class consists of arc-pairs $(\alpha, \beta)$ where $\alpha$ is the unique 2 -arc crossing $\beta$ and $\beta$ has length at least three. Without loss of generality we can restrict our analysis to the case $((i, i+2),(i+1, j)),(j>i+3)$.

- the arc $(i+1, j)$ is inflated to a 2 -stack. Then we have to insert at least one isolated vertex in either $[i, i+1]$ or $[i+1, i+2]$, see Fig. 11 . Therefore we have

$$
\mathcal{C}_{3}^{(\mathrm{a})}=\mathcal{R}^{2} \times\left(\operatorname{SEQ}(Z)^{2}-\mathcal{E}\right),
$$

with generating function

$$
\mathbf{C}_{3}^{(\mathrm{a})}(z)=z^{4}\left(\left(\frac{1}{1-z}\right)^{2}-1\right) .
$$

Note that the arc $(i, i+2)$ is not considered here, it can be inflated without any restrictions.

- the arc $(i+1, j)$ is inflated to an arbitrary $\dagger$-stem, see Fig. 11). Then

$$
\mathcal{C}_{3}^{(\mathrm{b})}=\left(\mathcal{M}-\mathcal{R}^{2}\right) \times \operatorname{SEQ}(\mathcal{Z})^{2},
$$

with generating function

$$
\mathbf{C}_{3}^{(\mathrm{b})}(z)=\left(\frac{\frac{z^{4}}{1-z^{2}}}{1-\frac{z^{4}}{1-z^{2}}\left(\frac{2 z}{1-z}+\left(\frac{z}{1-z}\right)^{2}\right)}-z^{4}\right) \cdot\left(\frac{1}{1-z}\right)^{2} .
$$

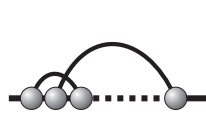

(a)
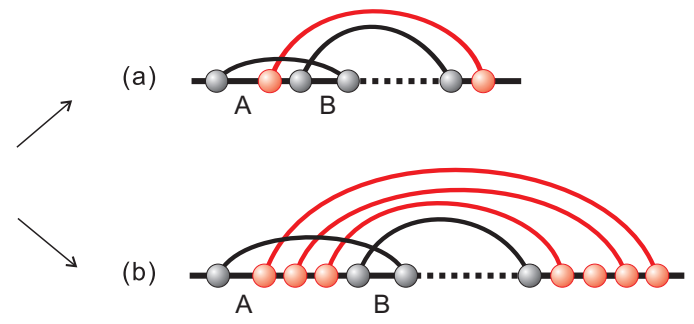

Fig. 11: $\mathbf{C}_{3}$-class: only one arc is inflated here and its inflation distinguishes two subcases. Arc-coloring as in Fig. 8

Consequently, this inflation process leads to a generating function

$$
\mathbf{C}_{3}(z)=\mathbf{C}_{3}^{(\mathrm{a})}(z)+\mathbf{C}_{3}^{(\mathrm{b})}(z) .
$$

Note that during inflation (a) and (b) only one of the two arcs of an $\mathbf{C}_{3}$-class element is being inflated.

- $\mathbf{C}_{4}$-class: this class consists of arc-triples $\left(\alpha_{1}, \beta, \alpha_{2}\right)$, where $\alpha_{1}$ and $\alpha_{2}$ are 2-arcs, respectively, that cross $\beta$. 
- $\beta$ is inflated to a 2-stack, see Fig. 12. Using similar arguments as in the case of $\mathbf{C}_{3}$-class, we arrive at

$$
\mathcal{C}_{4}^{(\mathrm{a})}=\mathcal{R}^{2} \times\left(\operatorname{SEQ}(Z)^{2}-\mathcal{E}\right) \times\left(\operatorname{SEQ}(Z)^{2}-\mathcal{E}\right)
$$

with generating function

$$
\mathbf{C}_{4}^{(\mathrm{a})}(z)=z^{4}\left(\left(\frac{1}{1-z}\right)^{2}-1\right)^{2}
$$

- the arc $\beta$ is inflated to an arbitrary †-stem, see Fig. 12,

$$
\mathcal{C}_{4}^{(\mathrm{b})}=\left(\mathcal{M}-\mathcal{R}^{2}\right) \times \operatorname{SEQ}(Z)^{4}
$$

with generating function

$$
\mathbf{C}_{4}^{(\mathrm{b})}(z)=\left(\frac{\frac{z^{4}}{1-z^{2}}}{1-\frac{z^{4}}{1-z^{2}}\left(\frac{2 z}{1-z}+\left(\frac{z}{1-z}\right)^{2}\right)}-z^{4}\right) \cdot\left(\frac{1}{1-z}\right)^{4}
$$

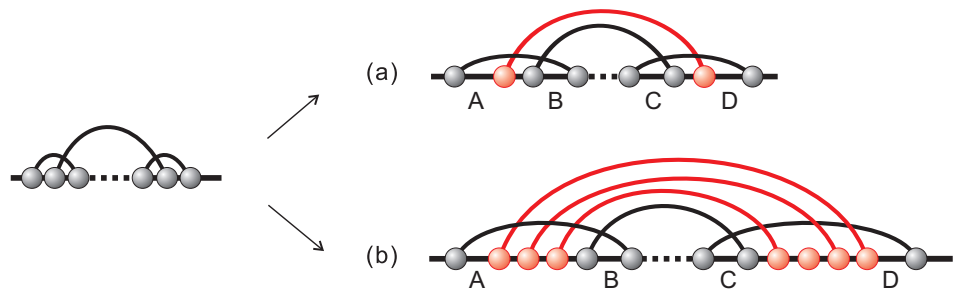

Fig. 12: $\mathbf{C}_{4}$-class: as for the inflation of $\mathbf{C}_{3}$ only the non 2-arc is inflated, distinguishing two subcases. Arc-coloring as in Fig. 8

Accordingly we arrive at

$$
\mathbf{C}_{4}(z)=\mathbf{C}_{4}^{(\mathrm{a})}(z)+\mathbf{C}_{4}^{(\mathrm{b})}(z)
$$

The inflation of any arc of $\gamma$ not considered in the previous steps follows the logic of Proposition 4. We observe that $\left(s-2 u_{2}-u_{3}-u_{4}\right)$ arcs of the shape $\gamma$ have not been considered. Furthermore, $\left(2 s+1-u_{1}-3 u_{2}-2 u_{3}-4 u_{4}\right)$ intervals were not considered for the insertion of isolated vertices. The inflation of these along the lines of Proposition 4 gives rise to the class

$$
\mathcal{S}=\mathcal{M}^{s-2 u_{2}-u_{3}-u_{4}} \times(\operatorname{SEQ}(Z))^{2 s+1-u_{1}-3 u_{2}-2 u_{3}-4 u_{4}},
$$

having the generating function

$$
\mathbf{S}(z)=\left(\frac{\frac{z^{4}}{1-z^{2}}}{1-\frac{z^{4}}{1-z^{2}}\left(\frac{2 z}{1-z}+\left(\frac{z}{1-z}\right)^{2}\right)}\right)^{s-2 u_{2}-u_{3}-u_{4}}\left(\frac{1}{1-z}\right)^{2 s+1-u_{1}-3 u_{2}-2 u_{3}-4 u_{4}} .
$$


Combining these observations Claim 1 follows.

Observing that $\mathbf{Q}_{\gamma_{1}}(z)=\mathbf{Q}_{\gamma_{2}}(z)$ for any $\gamma_{1}, \gamma_{2} \in \mathcal{J}_{k}(s, \vec{u})$, we have, according to eq. (5.4),

$$
\mathbf{Q}_{k}(z)=\sum_{s, \vec{u} \geqslant 0} i_{k}(s, \vec{u}) \mathbf{Q}_{\gamma}(z),
$$

where $\vec{u} \geqslant 0$ denotes $u_{i} \geqslant 0$ for $1 \leqslant i \leqslant 4$. Proposition 6 guarantees

$$
\begin{aligned}
& \sum_{s, \vec{u} \geqslant 0} i_{k}(s, \vec{u}) x^{s} y^{u_{1}} r^{u_{2}} w^{u_{3}} t^{u_{4}} \\
= & \frac{1+x}{1-(y-2) x+(2 w-r-1) x^{2}+(2 w-r-1) x^{3}} \times \\
& \mathbf{F}_{k}\left(\frac{x\left(1+(2 w-1) x+(t-1) x^{2}\right)}{\left(1-(y-2) x+(2 w-r-1) x^{2}+(2 w-r-1) x^{3}\right)^{2}}\right) .
\end{aligned}
$$

Setting $x=\varsigma_{0}(z), y=\varsigma_{1}(z), r=\varsigma_{2}(z), w=\varsigma_{3}(z), t=\varsigma_{4}(z)$, we arrive at

$$
\begin{aligned}
\mathbf{Q}_{k}(z)= & \frac{1-z^{2}+z^{4}}{1-z-z^{2}+z^{3}+2 z^{4}+z^{6}-z^{8}+z^{10}-z^{12}} \times \\
& \mathbf{F}_{k}\left(\frac{z^{4}\left(1-z^{2}-z^{4}+2 z^{6}-z^{8}\right)}{\left(1-z-z^{2}+z^{3}+2 z^{4}+z^{6}-z^{8}+z^{10}-z^{12}\right)^{2}}\right) .
\end{aligned}
$$

By Lemma $1, \mathbf{Q}_{k}(z)$ is $D$-finite. Pringsheim's Theorem [19] guarantees that $\mathbf{Q}_{k}(z)$ has a dominant real positive singularity $\gamma_{k}$. We verify that for $3 \leqslant k \leqslant 9, \gamma_{k}$ which is the unique solution of minimum modulus of the equation $\vartheta(z)=\rho_{k}^{2}$ is the unique dominant singularity of $\mathbf{Q}_{k}(z)$, and $\vartheta^{\prime}(z) \neq 0$. According to Proposition 2 we therefore have

$$
\mathrm{Q}_{k}(n) \sim c_{k} n^{-\left((k-1)^{2}+(k-1) / 2\right)}\left(\gamma_{k}^{-1}\right)^{n}, \quad \text { for some } c_{k}>0,
$$

and the proof of Theorem 3 is complete.

Remark 1. We remark that Theorem 3 does not hold for $k=2$, i.e. we cannot compute the generating function $\mathbf{Q}_{2}(z)$ via eq. (5.1). The reason is that Lemma 4 only holds for $k>2$ and indeed we find

$$
\mathbf{Q}_{2}(z) \neq \frac{1-z^{2}+z^{4}}{q(z)} \mathbf{F}_{2}\left(\frac{z^{4}\left(1-z^{2}-z^{4}+2 z^{6}-z^{8}\right)}{q(z)^{2}}\right) .
$$

However, the computation of the generating function $\mathbf{Q}_{2}(z)$ in Proposition 4 is based on Lemma 2, which does hold for $k=2$.

\section{Proof of Lemma 3 and Lemma 4}

\subsection{Proof of Lemma 3}

Proof. By construction, eq. (4.1) and eq. (4.2) hold. We next prove eq. (4.3). Choose a shape $\delta \in \mathcal{J}_{k}\left(s+1, u_{1}, u_{2}+1\right)$ and label exactly one of the $\left(u_{2}+1\right) \mathbf{C}_{2}$-elements. We 
denote the leftmost $\mathbf{C}_{2}$-arc (being a 2 -arc) by $\alpha$. Let $\mathcal{L}$ be the set of these labeled shapes, $\lambda$, then

$$
|\mathcal{L}|=\left(u_{2}+1\right) i_{k}\left(s+1, u_{1}, u_{2}+1\right) .
$$

We next observe that the removal of $\alpha$ results in either a shape or a matching. Let the elements of the former set be $\mathcal{L}_{1}$ and those of the latter $\mathcal{L}_{2}$. By construction,

$$
\mathcal{L}=\mathcal{L}_{1} \dot{\cup} \mathcal{L}_{2}
$$

Claim 1.

$$
\left|\mathcal{L}_{1}\right|=\left(u_{1}+1\right) i_{k}\left(s, u_{1}+1, u_{2}\right) .
$$

To prove Claim 1, we consider the labeled $\mathbf{C}_{2}$-element $(\alpha, \beta)$. Let $\mathcal{L}_{1}^{\alpha}$ be the set of shapes induced by removing $\alpha$. It is straightforward to verify that the removal of $\alpha$ can lead to only one additional $\mathbf{C}_{1}$-element, $\beta$. Therefore $\mathcal{L}_{1}$-shapes induce unique $\mathfrak{J}_{k}\left(s, u_{1}+1, u_{2}\right)$ shapes, having a labeled 1 -arc, $\beta$, see Fig. 13. This proves Claim 1.

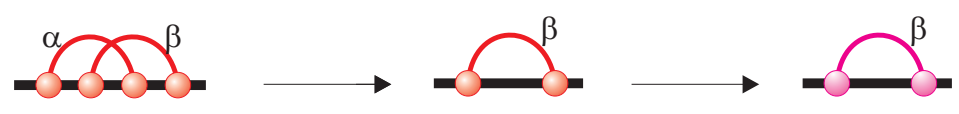

Fig. 13: The term $\left(u_{1}+1\right) i_{k}\left(s, u_{1}+1, u_{2}\right)$.

Claim 2.

$$
\left|\mathcal{L}_{2}\right|=\left(u_{1}+1\right) i_{k}\left(s-1, u_{1}+1, u_{2}\right) .
$$

To prove Claim 2, we consider $\mathcal{M}_{2}^{\alpha}$, the set of matchings, $\mu_{2}^{\alpha}$, obtained by removing $\alpha$. Such a matching contains exactly one stack of length two, $\left(\beta_{1}, \beta_{2}\right)$, where $\beta_{2}$ is nested in $\beta_{1}$. Let $\mathcal{L}_{2}^{\alpha}$ be the set of shapes induced by collapsing $\left(\beta_{1}, \beta_{2}\right)$ into $\beta_{2}$. We observe that $\alpha$ crosses $\beta_{2}$ and that $\beta_{2}$ becomes a 1-arc. Therefore, $\mathcal{L}_{2}$ is the set of labeled shapes, that induce unique $\mathfrak{J}_{k}\left(s-1, u_{1}+1, u_{2}\right)$-shapes having a labeled 1 -arc, $\beta_{2}$, see Fig. 14 . This proves Claim 2.

Combining Claim 1 and Claim 2 we derive eq. (4.3).

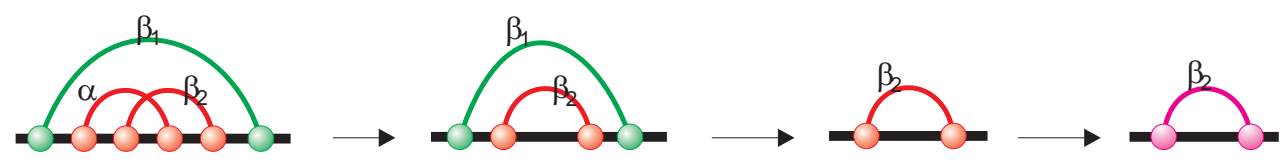

Fig. 14: The term $\left(u_{1}+1\right) i_{k}\left(s-1, u_{1}+1, u_{2}\right)$.

It remains to show (by induction on $s$ ) that the numbers $i_{k}\left(s, u_{1}, u_{2}\right)$ can be uniquely derived from eq. (4.1), eq. (4.2) and eq. (4.3), whence the lemma. 


\subsection{Proof of Lemma 4}

Proof. By construction, eq. (4.11) and eq. (4.12) hold. We next prove eq. (4.13).

Choose a shape $\delta \in \mathcal{J}_{k}\left(s+1, u_{1}, u_{2}, u_{3}+1, u_{4}\right)$ and label exactly one of the $\left(u_{3}+1\right) \mathbf{C}_{3^{-}}$ elements containing a unique 2 -arc, $\alpha$. We denote the set of these labeled shapes, $\lambda$, by L. Clearly

$$
|\mathcal{L}|=\left(u_{3}+1\right) i_{k}\left(s+1, u_{1}, u_{2}, u_{3}+1, u_{4}\right) .
$$

We observe that the removal of $\alpha$ results in either a shape $\left(\mathcal{L}_{1}\right)$ or a matching $\left(\mathcal{L}_{2}\right)$, i.e. we have

$$
\mathcal{L}=\mathcal{L}_{1} \dot{\cup} \mathcal{L}_{2}
$$

Claim 1.

$$
\begin{aligned}
\left|\mathcal{L}_{1}\right|= & 2\left(u_{3}+1\right) i_{k}\left(s, u_{1}, u_{2}, u_{3}+1, u_{4}\right)+ \\
& 4\left(u_{4}+1\right) i_{k}\left(s, u_{1}, u_{2}, u_{3}-1, u_{4}+1\right)+ \\
& \left(2\left(s-u_{1}-2 u_{2}-2 u_{3}-3 u_{4}\right)\right) i_{k}\left(s, u_{1}, u_{2}, u_{3}, u_{4}\right) .
\end{aligned}
$$

To prove Claim 1, we consider the labeled $\mathbf{C}_{3}$-element of a $\mathcal{L}_{1}$-shape, $(\alpha, \beta)$. We set $\mathcal{L}_{1}^{\alpha}$ to be the set of shapes induced by removing $\alpha$ and denote the resulting shapes by $\lambda_{1}^{\alpha}$. By construction a $\lambda_{1}^{\alpha}$-shape cannot contain any additional $\mathbf{C}_{1^{-}}$or $\mathbf{C}_{2}$-elements, see Fig. 16 . Clearly, the removal of $\alpha$ can lead to at most one additional $\mathbf{C}_{i}$-element, whence

$$
\mathcal{L}_{1}=\mathcal{L}_{1}^{\mathbf{C}_{3}} \dot{\cup} \mathcal{L}_{1}^{\mathbf{C}_{4}} \dot{\cup} \mathcal{L}_{1}^{0},
$$

where $\mathcal{L}_{1}^{\mathbf{C}_{i}}, i=3,4$ denotes the set of labeled shapes, $\lambda \in \mathcal{L}_{1}$, that induce a unique shape having a labeled $\mathbf{C}_{i}$-element containing $\beta$ and $\mathcal{L}_{1}^{0}$ the set of those shapes, in which there exists no such $\mathbf{C}_{i}$-element.

We first prove

$$
\left|\mathcal{L}_{1}^{\mathbf{C}_{3}}\right|=2\left(u_{3}+1\right) i_{k}\left(s, u_{1}, u_{2}, u_{3}+1, u_{4}\right) .
$$

Indeed, in order to generate a labeled $\mathbf{C}_{3}$-element by $\alpha$-removal from $\mathcal{L}_{1}$-shape, $\beta$ has to become a 2 -arc in a labeled $\mathbf{C}_{3}$-element of a $\mathfrak{J}_{k}\left(s, u_{1}, u_{2}, u_{3}+1, u_{4}\right)$-shape, see Fig. 17. Next we prove

$$
\left|\mathcal{L}_{1}^{\mathbf{C}_{4}}\right|=4\left(u_{4}+1\right) i_{k}\left(s, u_{1}, u_{2}, u_{3}-1, u_{4}+1\right) .
$$

Indeed in order to generate a labeled $\mathbf{C}_{4}$-element by $\alpha$-removal from $\mathcal{L}_{1}$-shape, $\beta$ has to become a 2 -arc in a labeled $\mathbf{C}_{4}$-element of a $\mathfrak{J}_{k}\left(s, u_{1}, u_{2}, u_{3}-1, u_{4}+1\right)$-shape. We display all possible scenarios in Fig. 18.

Otherwise $\beta$ becomes simply a labeled arc in a $\mathfrak{J}_{k}\left(s, u_{1}, u_{2}, u_{3}, u_{4}\right)$-shape, which is not contained in any $\mathbf{C}_{i}$-element, whence

$$
\left|\mathcal{L}_{1}^{0}\right|=2\left(s-u_{1}-2 u_{2}-2 u_{3}-3 u_{4}\right) i_{k}\left(s, u_{1}, u_{2}, u_{3}, u_{4}\right)
$$




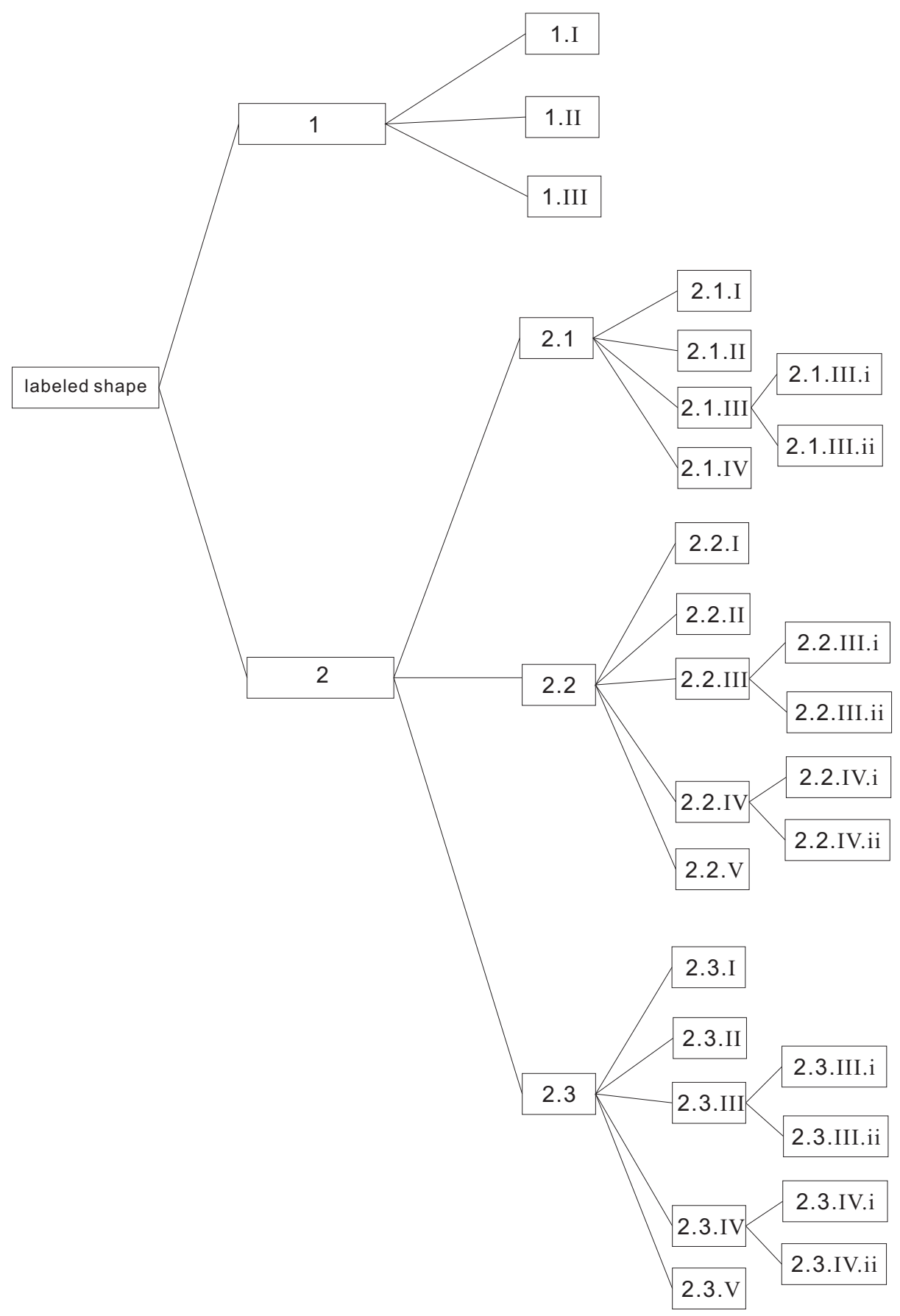

Fig. 15: Accounting: the scenarious arising from the removal of $\alpha$ from a labeled $\mathbf{C}_{3^{-}}$ element of a $\mathcal{J}_{k}\left(s+1, u_{1}, u_{2}, u_{3}+1, u_{4}\right)$-shape. 


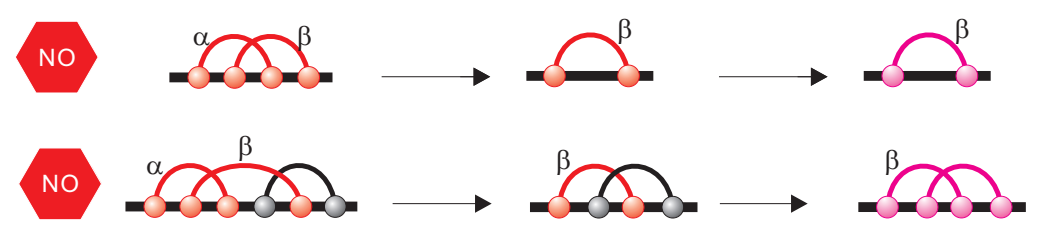

Fig. 16: The removal of $\alpha$ cannot give rise to additional $\mathbf{C}_{1-}$ or $\mathbf{C}_{2}$-elements.

1.I

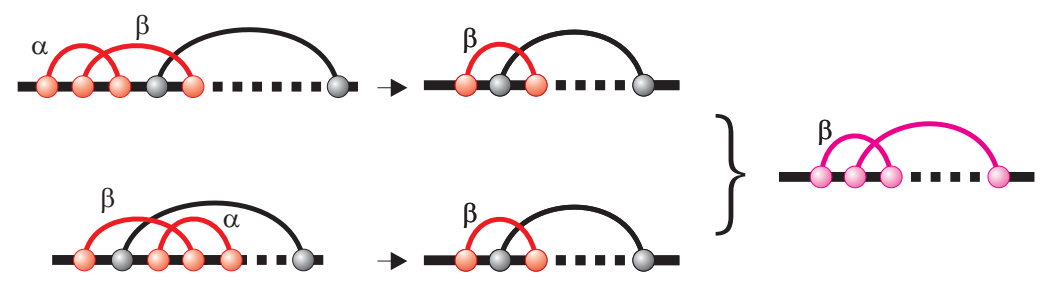

Fig. 17: We illustrate the effect of the removal of $\alpha$ when inducing a labeled $\mathbf{C}_{3}$-element.
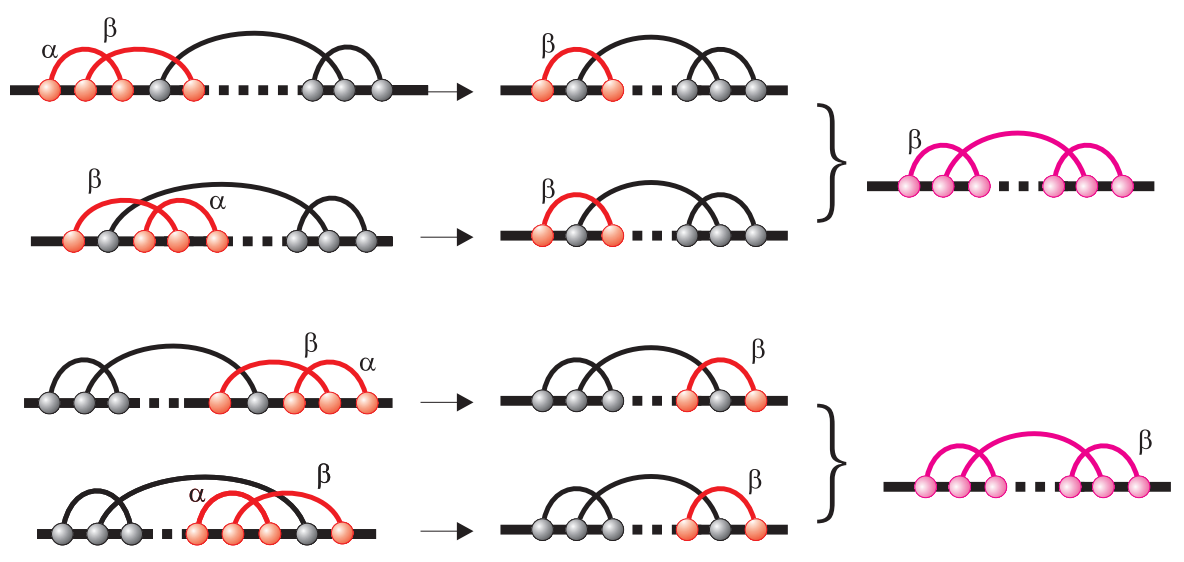

Fig. 18: We illustrate the effect of the removal of $\alpha$ with when inducing a labeled $\mathbf{C}_{4}$-element. 
and Claim 1 follows.

We next consider $\mathcal{L}_{2}$. Let $\mathcal{M}_{2}^{\alpha}$ be the set of matchings, $\mu_{2}^{\alpha}$, obtained by removing $\alpha$. Claim 2. Let $\left(\beta_{1}, \ldots, \beta_{\ell}\right)$ denote a $\mu_{2}^{\alpha}$-stack $\left(\left(\beta_{1}, \ldots, \beta_{\ell}\right) \prec \mu_{2}^{\alpha}\right)$. Then we have

$$
\mathcal{L}_{2}=\mathcal{L}_{2,1} \dot{\cup} \mathcal{L}_{2,2} \dot{\cup} \mathcal{L}_{2,3}
$$

where

$$
\begin{aligned}
& \mathcal{L}_{2,1}=\left\{\lambda \in \mathcal{L}_{2} \mid \alpha, \beta_{i} \in \lambda, i=1,2 ;\left(\beta_{1}, \beta_{2}\right) \prec \mu_{2}^{\alpha} ; \alpha \text { crosses } \beta_{2}\right\}, \\
& \mathcal{L}_{2,2}=\left\{\lambda \in \mathcal{L}_{2} \mid \alpha, \beta_{i} \in \lambda, i=1,2 ;\left(\beta_{1}, \beta_{2}\right) \prec \mu_{2}^{\alpha} ; \alpha \text { crosses } \beta_{1}\right\}, \\
& \mathcal{L}_{2,3}=\left\{\lambda \in \mathcal{L}_{2} \mid \alpha, \beta_{i} \in \lambda, i=1,2,3 ;\left(\beta_{1}, \beta_{2}, \beta_{3}\right) \prec \mu_{2}^{\alpha} ; \alpha \text { crosses } \beta_{2}\right\} .
\end{aligned}
$$

To prove Claim 2, it suffices to observe that a $\mathcal{M}_{2}^{\alpha}$-matching contains exactly one stack of length either two or three. Now, eq. (6.3) immediately follows by inspection of Figure 19. Claim 2.1
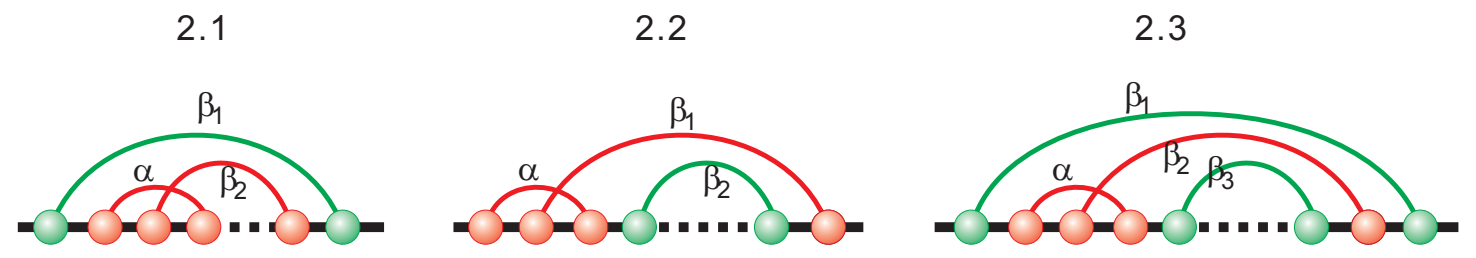

Fig. 19: $\mathcal{L}_{2}$ and $\mathcal{N}_{2}^{\alpha}$ : how stacks arise by the removal of $\alpha$.

$$
\begin{aligned}
\left|\mathcal{L}_{2,1}\right| & =4\left(u_{2}+1\right) i_{k}\left(s-1, u_{1}, u_{2}+1, u_{3}, u_{4}\right) \\
& +4\left(u_{3}+1\right) i_{k}\left(s-1, u_{1}, u_{2}, u_{3}+1, u_{4}\right) \\
& +\left[4\left(u_{4}+1\right) i_{k}\left(s-1, u_{1}, u_{2}, u_{3}-1, u_{4}+1\right)\right. \\
& \left.+2\left(u_{4}+1\right) i_{k}\left(s-1, u_{1}, u_{2}, u_{3}, u_{4}+1\right)\right] \\
& \left.+2\left((s-1)-u_{1}-2 u_{2}-2 u_{3}-3 u_{4}\right)\right) i_{k}\left(s-1, u_{1}, u_{2}, u_{3}, u_{4}\right) .
\end{aligned}
$$

To prove Claim 2.1, let $\mathcal{M}_{2,1}^{\alpha}$ be the set of matchings induced by removing $\alpha$ from a $\mathcal{L}_{2,1^{-}}$ shape. We set $\mathcal{L}_{2,1}^{\alpha}$ to be the set of shapes induced by collapsing the unique $\mathcal{M}_{2,1}^{\alpha}$-stack of length two into the arc $\beta_{2}$. Clearly, such a shape cannot exhibit any additional $\mathbf{C}_{1^{-}}$ elements, see Fig. 20.

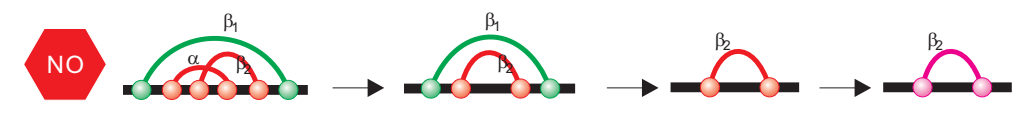

Fig. 20: $\lambda_{2,1}^{\alpha}$ cannot exhibit any additional $\mathbf{C}_{1}$-elements.

Since the removal of $\alpha$ and subsequent stack-collapse can lead to at most one new $\mathbf{C}_{i}$ element, we have

$$
\mathcal{L}_{2,1}=\mathcal{L}_{2,1}^{\mathbf{C}_{2}} \dot{\cup} \mathcal{L}_{2,1}^{\mathbf{C}_{3}} \dot{\cup} \mathcal{L}_{2,1}^{\mathbf{C}_{4}} \dot{\cup} \mathcal{L}_{2,1}^{0}
$$


where $\mathcal{L}_{2,1}^{\mathbf{C}_{i}}, i=2,3,4$ denotes the set of labeled shapes, $\lambda \in \mathcal{L}_{2,1}$, that induce unique shapes having a labeled $\mathbf{C}_{i}$-element containing $\beta_{2}$ and $\mathcal{L}_{2,1}^{0}$ denotes those in which there exists no $\mathbf{C}_{i}$-element containing $\beta_{2}$.

We first prove

$$
\left|\mathcal{L}_{2,1}^{\mathbf{C}_{2}}\right|=4\left(u_{2}+1\right) i_{k}\left(s-1, u_{1}, u_{2}+1, u_{3}, u_{4}\right) .
$$

Indeed, in order to generate a labeled $\mathbf{C}_{2}$-element via $\alpha$-removal and subsequent stack-

\subsection{I}

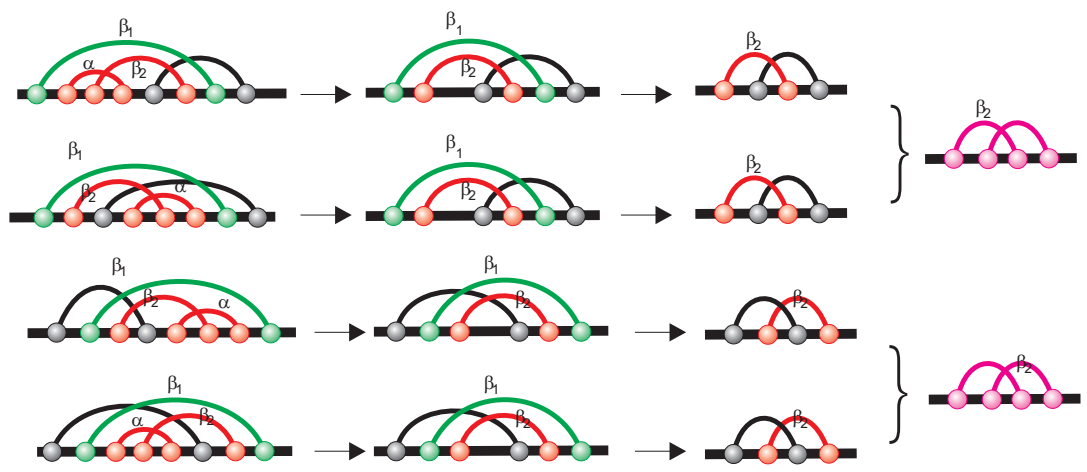

Fig. 21: In $\mathcal{L}_{2,1}$ : the removal of $\alpha$ and subsequent collapse of the unique stack of length two in $\mathcal{N}_{2,1}^{\alpha}$, generating a labeled $\mathbf{C}_{2}$-element (pink).

collapse from a $\mathcal{L}_{2,1}$-shape, $\beta_{2}$ has to become a 2 -arc in a $\mathbf{C}_{2}$-element of a $\mathcal{J}_{k}\left(s-1, u_{1}, u_{2}+\right.$ $1, u_{3}, u_{4}$ )-shape. We display all possible scenarios in Fig. 21.

Next we prove

$$
\left|\mathcal{L}_{2,1}^{\mathbf{C}_{3}}\right|=4\left(u_{3}+1\right) i_{k}\left(s-1, u_{1}, u_{2}, u_{3}+1, u_{4}\right) .
$$

In order to generate a labeled $\mathbf{C}_{3}$-element by $\alpha$-removal and collapsing the unique stack of length two from a $\mathcal{L}_{2,1}$-shape, $\beta_{2}$ has to become a 2 -arc or an arc uniquely crossing a 2 -arc in a $\mathbf{C}_{3}$-element of a $\mathcal{J}_{k}\left(s-1, u_{1}, u_{2}, u_{3}+1, u_{4}\right)$-shape. We display all possible scenarios in Fig. 22.

Third we prove

$$
\left|\mathcal{L}_{2,1}^{\mathbf{C}_{4}}\right|=4\left(u_{4}+1\right) i_{k}\left(s-1, u_{1}, u_{2}, u_{3}-1, u_{4}+1\right)+2\left(u_{4}+1\right) i_{k}\left(s-1, u_{1}, u_{2}, u_{3}, u_{4}+1\right) .
$$

In order to generate a labeled $\mathbf{C}_{4}$-element by $\alpha$-removal and collapsing the unique stack of length two from a $\mathcal{L}_{2,1}$-shape, $\beta_{2}$ has to become either a 2 -arc in a labeled $\mathbf{C}_{4}$-element of a $\mathcal{J}_{k}\left(s-1, u_{1}, u_{2}, u_{3}-1, u_{4}+1\right)$-shape or an arc that crosses two 2-arcs in a labeled $\mathbf{C}_{4}$-element of a $\mathcal{J}_{k}\left(s-1, u_{1}, u_{2}, u_{3}, u_{4}+1\right)$-shape. We display all possible scenarios in Fig. 23 and Fig. 24.

Otherwise, $\beta_{2}$ becomes a labeled arc in a $\mathcal{J}_{k}\left(s-1, u_{1}, u_{2}, u_{3}, u_{4}\right)$-shape, which is not contained in any $\mathbf{C}_{i}$-element. Thus

$$
\left.\left|\mathcal{L}_{2,1}^{0}\right|=2\left((s-1)-u_{1}-2 u_{2}-2 u_{3}-3 u_{4}\right)\right) i_{k}\left(s-1, u_{1}, u_{2}, u_{3}, u_{4}\right),
$$




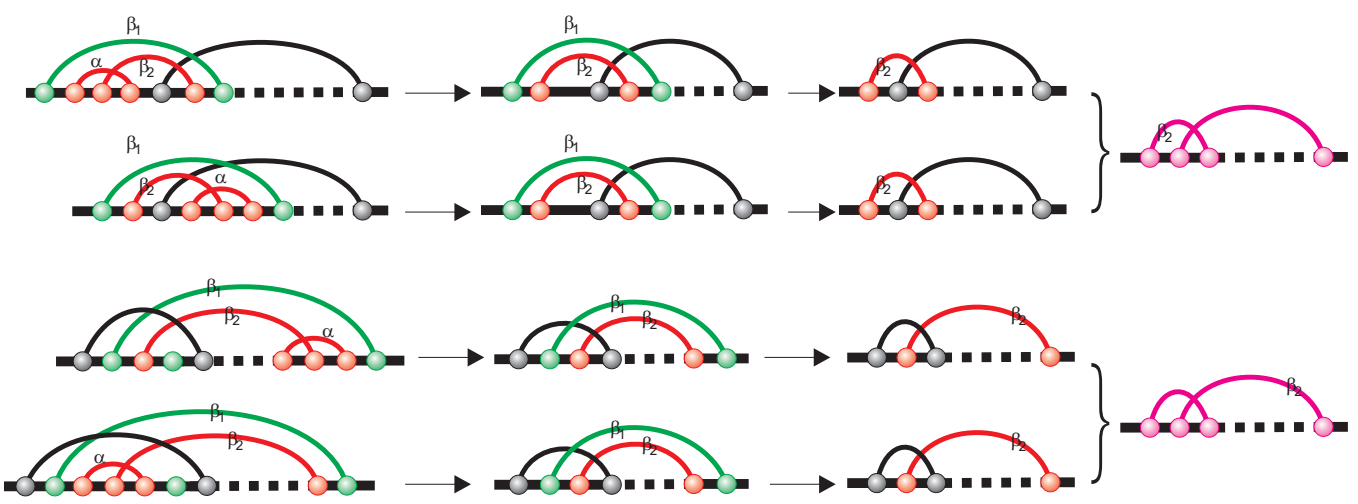

Fig. 22: In $\mathcal{L}_{2,1}$ : the removal of $\alpha$ and collapsing the unique stack of length two in $\mathcal{N}_{2,1}^{\alpha}$, generating a labeled $\mathbf{C}_{3}$-element (pink).

\subsection{III.i}

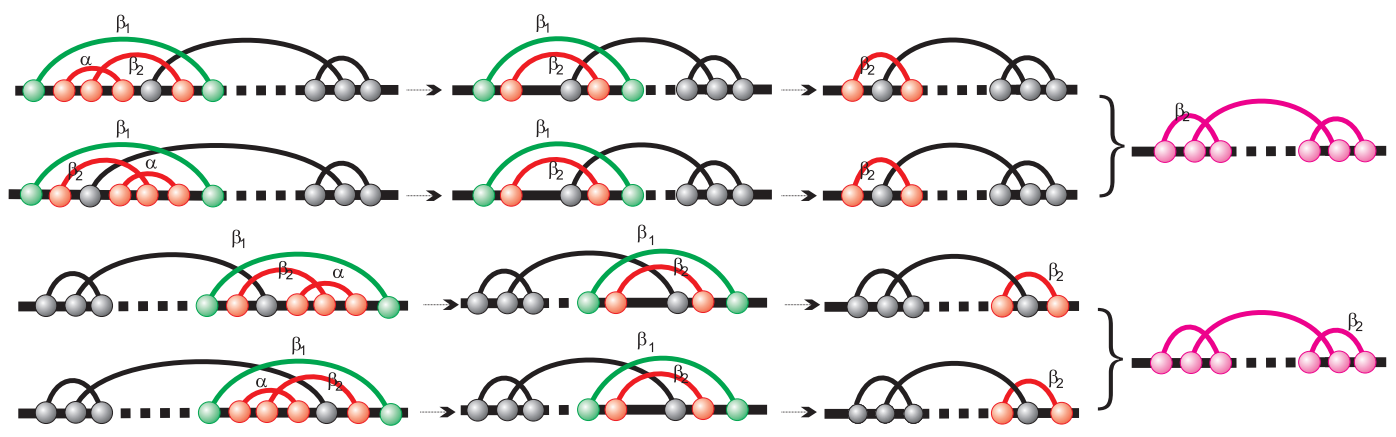

Fig. 23: How to derive a labeled $\mathbf{C}_{4}$-element: first scenario.

\subsection{III.ii}

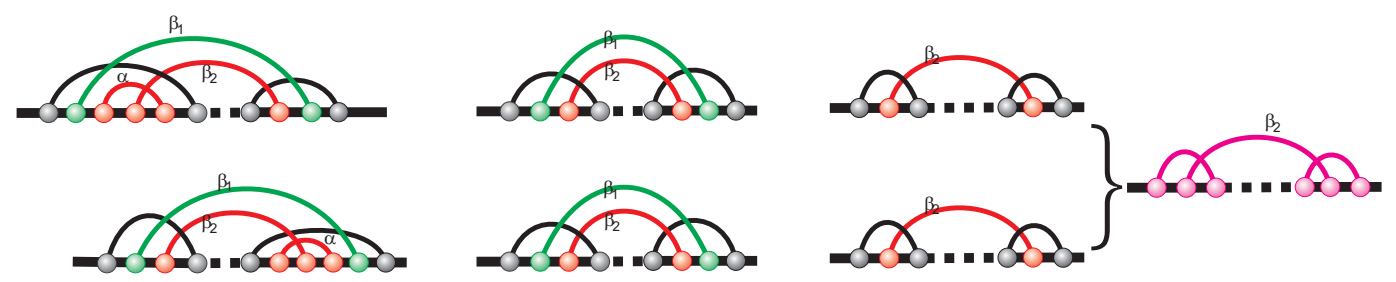

Fig. 24: How to derive a labeled $\mathbf{C}_{4}$-element:second scenario. 
from which Claim 2.1 follows.

Claim 2.2.

$$
\begin{aligned}
\left|\mathcal{L}_{2,2}\right| & =2 u_{1} i_{k}\left(s-1, u_{1}, u_{2}, u_{3}, u_{4}\right) \\
& +4\left(u_{2}+1\right) i_{k}\left(s-1, u_{1}, u_{2}+1, u_{3}-1, u_{4}\right) \\
& +\left[2 u_{3} i_{k}\left(s-1, u_{1}, u_{2}, u_{3}, u_{4}\right)\right. \\
& \left.+2\left(u_{3}+1\right) i_{k}\left(s-1, u_{1}, u_{2}, u_{3}+1, u_{4}\right)\right] \\
& +\left[4 u_{4} i_{k}\left(s-1, u_{1}, u_{2}, u_{3}, u_{4}\right)\right. \\
& \left.+2\left(u_{4}+1\right) i_{k}\left(s-1, u_{1}, u_{2}, u_{3}, u_{4}+1\right)\right] \\
& \left.+2\left((s-1)-u_{1}-2 u_{2}-2 u_{3}-3 u_{4}\right)\right) i_{k}\left(s-1, u_{1}, u_{2}, u_{3}, u_{4}\right) .
\end{aligned}
$$

In order to prove Claim 2.2, we consider $\mathcal{M}_{2,2}^{\alpha}$, the set of matchings induced by removing $\alpha$ from a $\mathcal{L}_{2,2}$-shape. We set $\mathcal{L}_{2,2}^{\alpha}$ to be the set of shapes induced by collapsing the unique $\mathcal{M}_{2,2}^{\alpha}$-stack of length two into $\beta_{2}$. The removal of $\alpha$ and subsequent collapse can only lead to at most one additional $\mathbf{C}_{i}$-element, whence

$$
\mathcal{L}_{2,2}=\mathcal{L}_{2,2}^{\mathbf{C}_{1}} \dot{\cup} \mathcal{L}_{2,2}^{\mathbf{C}_{2}} \dot{\cup} \mathcal{L}_{2,2}^{\mathbf{C}_{3}} \dot{\cup} \mathcal{L}_{2,2}^{\mathbf{C}_{4}} \dot{\cup} \mathcal{L}_{2,2}^{0},
$$

using analogous notation and reasoning as in the proof of Claim 2.1.

We first prove

$$
\left|\mathcal{L}_{2,2}^{\mathrm{C}_{1}}\right|=2 u_{1} i_{k}\left(s-1, u_{1}, u_{2}, u_{3}, u_{4}\right) .
$$

In order to generate a labeled $\mathbf{C}_{1}$-element by $\alpha$-removal from a $\mathcal{L}_{2,2}$-shape and collapsing

\subsection{I}

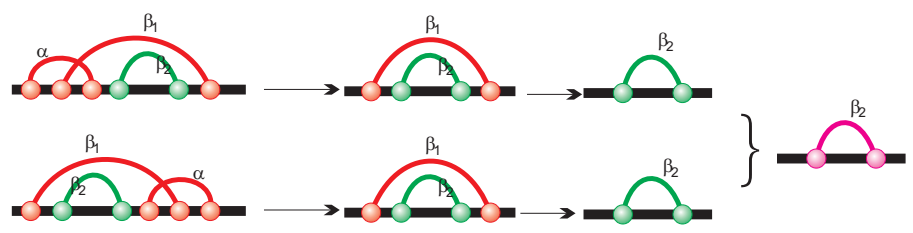

Fig. 25: Removal of $\alpha$ in $\mathcal{L}_{2,2}^{\alpha}$ and subsequent collapsing of the unique stack in $\mathcal{M}_{2,2}^{\alpha}$, generating a labeled $\mathbf{C}_{1}$-element.

the unique stack of length two, we need $\beta_{2}$ to be a 1 -arc in a $\mathfrak{J}_{k}\left(s-1, u_{1}, u_{2}, u_{3}, u_{4}\right)$-shape, see Figure 25. Note that this operation only transfers labels but generates no new 1-arcs. Next we prove

$$
\left|\mathcal{L}_{2,2}^{\mathbf{C}_{2}}\right|=4\left(u_{2}+1\right) i_{k}\left(s-1, u_{1}, u_{2}+1, u_{3}-1, u_{4}\right) .
$$

In order to generate a labeled $\mathbf{C}_{2}$-element by $\alpha$-removal from a $\mathcal{L}_{2,1}$-shape and collapsing the unique stack of length two, $\beta_{2}$ has to become a 2 -arc in a labeled $\mathbf{C}_{2}$-element of $\mathfrak{J}_{k}\left(s-1, u_{1}, u_{2}+1, u_{3}-1, u_{4}\right)$. We display all possible scenarios in Fig. 26. Third we prove

$$
\left|\mathcal{L}_{2,2}^{\mathbf{C}_{3}}\right|=2 u_{3} i_{k}\left(s-1, u_{1}, u_{2}, u_{3}, u_{4}\right)+2\left(u_{3}+1\right) i_{k}\left(s-1, u_{1}, u_{2}, u_{3}+1, u_{4}\right) .
$$




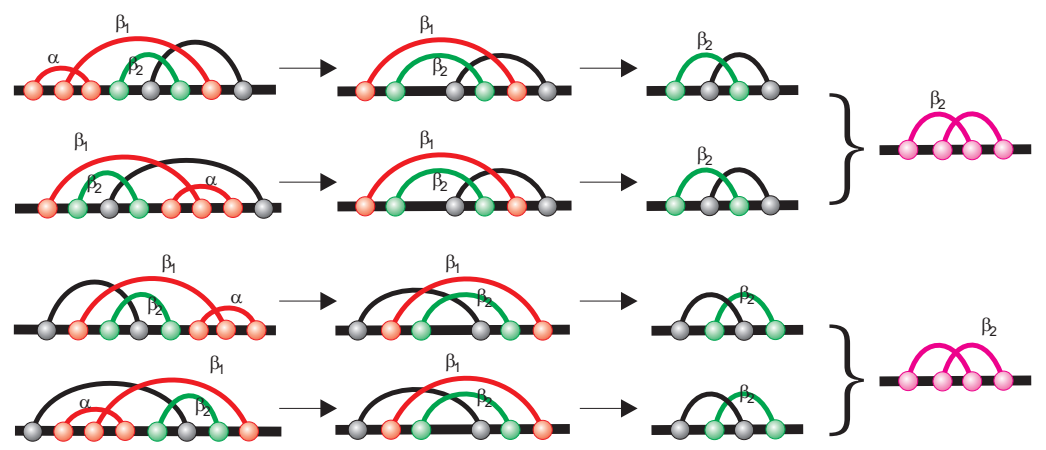

Fig. 26: Removal of $\alpha$ (red arc) in $\mathcal{L}_{2,2}^{\alpha}$ and collapsing the unique stack of length two in $\mathcal{M}_{2,2}^{\alpha}$, generating a labeled $\mathbf{C}_{2}$-element.

\subsection{III.i}

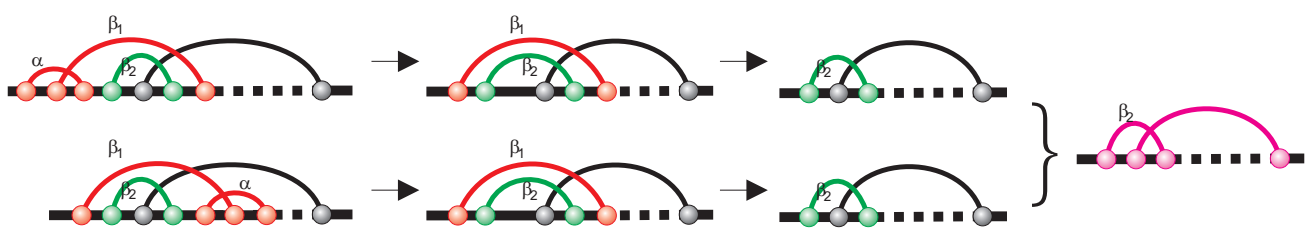

Fig. 27: The term $2 u_{3} i_{k}\left(s-1, u_{1}, u_{2}, u_{3}, u_{4}\right)$ : removal of $\alpha$ in $\mathcal{L}_{2,2}^{\alpha}$ and collapsing the unique stack of length two in $\mathcal{M}_{2,2}^{\alpha}$, generating a labeled $\mathbf{C}_{3}$-element.

\subsection{III.ii}

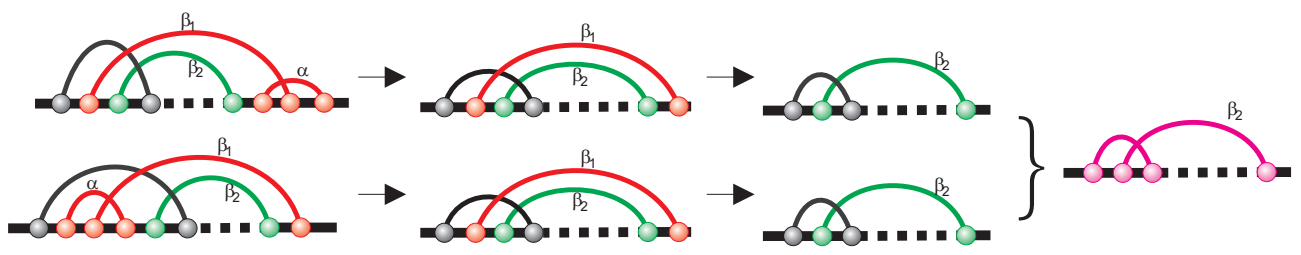

Fig. 28: The term $2\left(u_{3}+1\right) i_{k}\left(s-1, u_{1}, u_{2}, u_{3}+1, u_{4}\right)$. 
In order to generate a labeled $\mathbf{C}_{3}$-element by $\alpha$-removal from a $\mathcal{L}_{2,2}$-shape and collapse of the resulting unique stack, $\beta_{2}$ has to become either a 2 -arc in a labeled $\mathbf{C}_{3}$-element of a $\mathcal{J}_{k}\left(s-1, u_{1}, u_{2}, u_{3}, u_{4}\right)$-shape or an arc uniquely crossing the 2 -arc in a labeled $\mathbf{C}_{3}$-element of a $\mathcal{J}_{k}\left(s-1, u_{1}, u_{2}, u_{3}+1, u_{4}\right)$-shape. We display all possible scenarios in Fig. 27 and Fig.28.

Fourth we prove

$$
\left|\mathcal{L}_{2,2}^{\mathbf{C}_{4}}\right|=4 u_{4} i_{k}\left(s-1, u_{1}, u_{2}, u_{3}, u_{4}\right)+2\left(u_{4}+1\right) i_{k}\left(s-1, u_{1}, u_{2}, u_{3}, u_{4}+1\right) .
$$

In order to generate a labeled $\mathbf{C}_{4}$-element, $\beta_{2}$ has to become either a 2 -arc in a labeled

\subsection{IV.i}

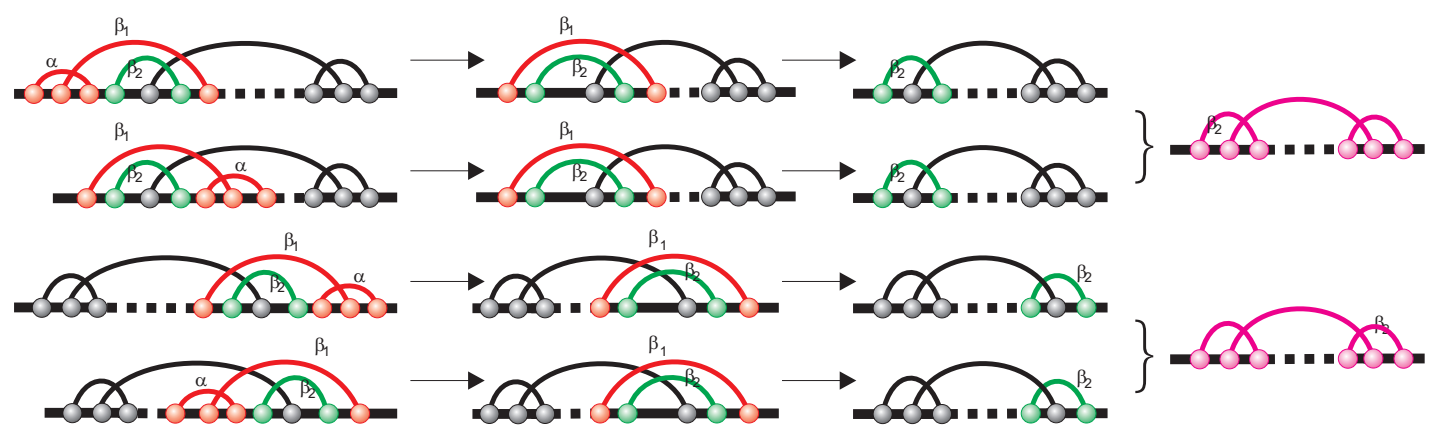

Fig. 29: The term $4 u_{4} i_{k}\left(s-1, u_{1}, u_{2}, u_{3}, u_{4}\right)$.

\subsection{IV.ii}
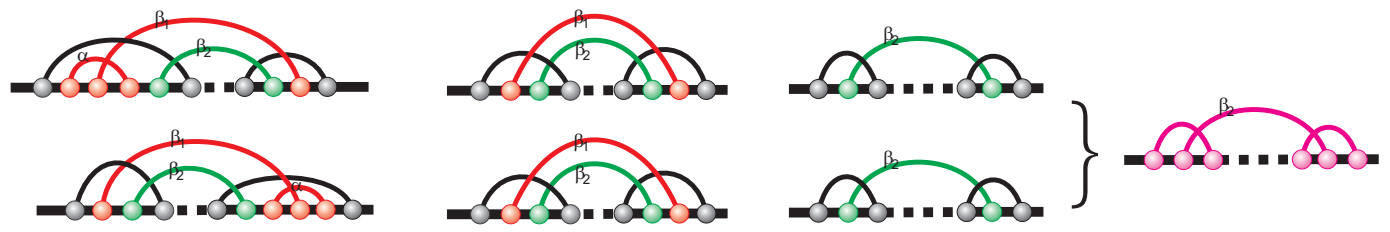

Fig. 30: The term $2\left(u_{4}+1\right) i_{k}\left(s-1, u_{1}, u_{2}, u_{3}, u_{4}+1\right)$.

$\mathbf{C}_{4}$-element of a $\mathcal{J}_{k}\left(s-1, u_{1}, u_{2}, u_{3}, u_{4}\right)$-shape or an arc uniquely crossing two 2-arcs in a labeled $\mathbf{C}_{4}$-element of a $\mathcal{J}_{k}\left(s-1, u_{1}, u_{2}, u_{3}, u_{4}+1\right)$-shape. We display all possible scenarios in Fig. 29 and Fig. 30.

It remains to observe that $\beta_{2}$ otherwise becomes a labeled arc in a $\mathcal{J}_{k}\left(s-1, u_{1}, u_{2}, u_{3}, u_{4}\right)$ shape, which is not contained in any $\mathbf{C}_{i}$-element. Thus

$$
\left.\left|\mathcal{L}_{2,2}^{0}\right|=2\left((s-1)-u_{1}-2 u_{2}-2 u_{3}-3 u_{4}\right)\right) i_{k}\left(s-1, u_{1}, u_{2}, u_{3}, u_{4}\right)
$$

and Claim 2.2 follows. 
Claim 2.3

$$
\begin{aligned}
\left|\mathcal{L}_{2,3}\right| & =2 u_{1} i_{k}\left(s-2, u_{1}, u_{2}, u_{3}, u_{4}\right) \\
& +4\left(u_{2}+1\right) i_{k}\left(s-2, u_{1}, u_{2}+1, u_{3}-1, u_{4}\right) \\
& +\left[2 u_{3} i_{k}\left(s-2, u_{1}, u_{2}, u_{3}, u_{4}\right)\right. \\
& \left.+2\left(u_{3}+1\right) i_{k}\left(s-2, u_{1}, u_{2}, u_{3}+1, u_{4}\right)\right] \\
& +\left[4 u_{4} i_{k}\left(s-2, u_{1}, u_{2}, u_{3}, u_{4}\right)\right. \\
& \left.+2\left(u_{4}+1\right) i_{k}\left(s-2, u_{1}, u_{2}, u_{3}, u_{4}+1\right)\right] \\
& \left.+2\left((s-2)-u_{1}-2 u_{2}-2 u_{3}-3 u_{4}\right)\right) i_{k}\left(s-2, u_{1}, u_{2}, u_{3}, u_{4}\right) .
\end{aligned}
$$

Let $\mathcal{M}_{2,3}^{\alpha}$ be the set of matchings induced by removing $\alpha$ from a $\mathcal{L}_{2,3}$-shape. Let $\mathcal{L}_{2,3}^{\alpha}$ denote the set of shapes induced by collapsing the unique $\mathcal{M}_{2,3}^{\alpha}$-stack of length three into the arc $\beta_{3}$. The removal of $\alpha$ and subsequent stack-collapse can only lead to at most one additional $\mathbf{C}_{i}(i=1,2,3,4)$ element, whence

$$
\mathcal{L}_{2,3}=\mathcal{L}_{2,3}^{\mathbf{C}_{1}} \dot{\cup} \mathcal{L}_{2,3}^{\mathbf{C}_{2}} \dot{\cup} \mathcal{L}_{2,3}^{\mathbf{C}_{3}} \dot{\cup} \mathcal{L}_{2,3}^{\mathbf{C}_{4}} \dot{\cup} \mathcal{L}_{2,3}^{0},
$$

where $\mathcal{L}_{2,3}^{\mathbf{C}_{i}}$ denotes the set of labeled shapes, $\lambda \in \mathcal{L}_{2,3}$, that induce unique shapes having a labeled $\mathbf{C}_{i}$-element containing $\beta_{3}$ and $\mathcal{L}_{2,3}^{0}$ denotes those shapes in which there exists no such $\mathbf{C}_{i}$-element.

We first note

$$
\left|\mathcal{L}_{2,3}^{\mathbf{C}_{1}}\right|=2 u_{1} i_{k}\left(s-2, u_{1}, u_{2}, u_{3}, u_{4}\right)
$$

see Fig. 31.

Next we observe

\subsection{I}

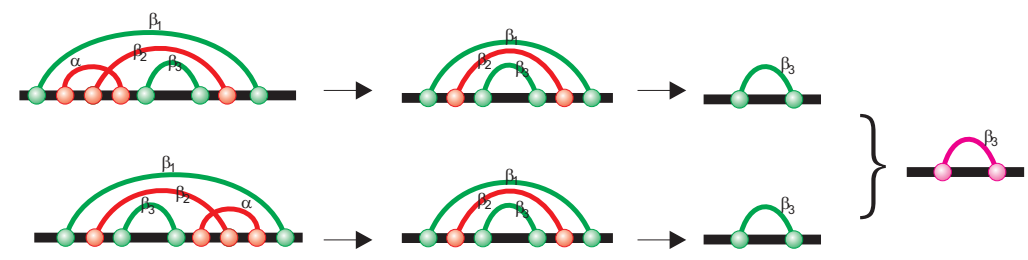

Fig. 31: The term $2 u_{1} i_{k}\left(s-2, u_{1}, u_{2}, u_{3}, u_{4}\right)$.

$$
\left|\mathcal{L}_{2,3}^{\mathbf{C}_{2}}\right|=4\left(u_{2}+1\right) i_{k}\left(s-2, u_{1}, u_{2}+1, u_{3}-1, u_{4}\right)
$$

see Fig. 32.

Third we verify

$$
\left|\mathcal{L}_{2,3}^{\mathbf{C}_{3}}\right|=2 u_{3} i_{k}\left(s-2, u_{1}, u_{2}, u_{3}, u_{4}\right)+2\left(u_{3}+1\right) i_{k}\left(s-2, u_{1}, u_{2}, u_{3}+1, u_{4}\right),
$$

see Fig. 33 and Fig. 34.

Fourth we note 


\subsection{II}

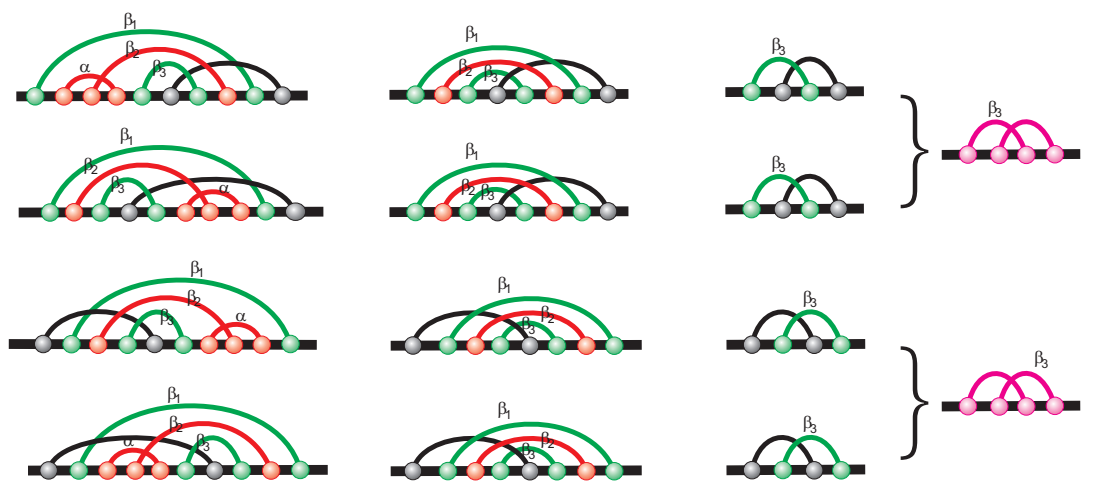

Fig. 32: The term $4\left(u_{2}+1\right) i_{k}\left(s-2, u_{1}, u_{2}+1, u_{3}-1, u_{4}\right)$.

\subsection{III.i}

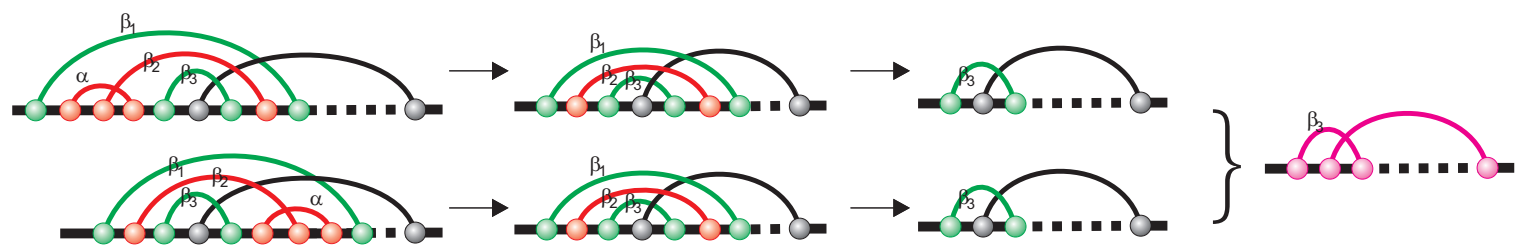

Fig. 33: The term $2 u_{3} i_{k}\left(s-2, u_{1}, u_{2}, u_{3}, u_{4}\right)$.

\subsection{III.ii}

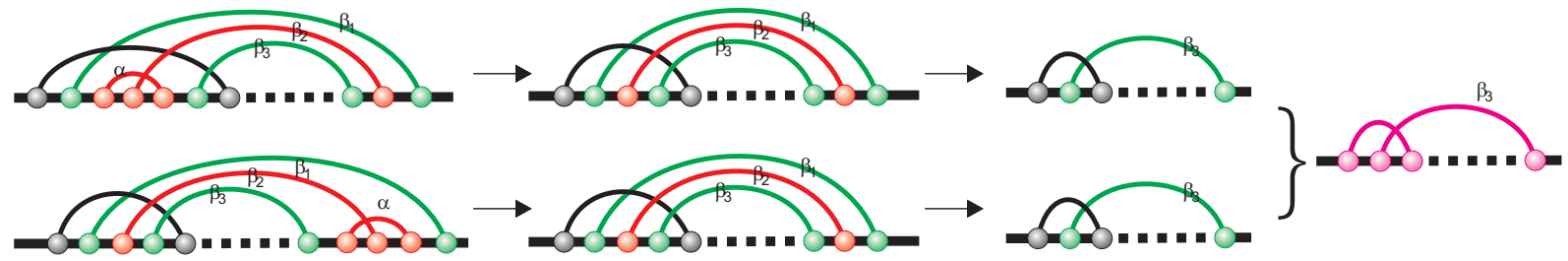

Fig. 34: The term $2\left(u_{3}+1\right) i_{k}\left(s-2, u_{1}, u_{2}, u_{3}+1, u_{4}\right)$. 


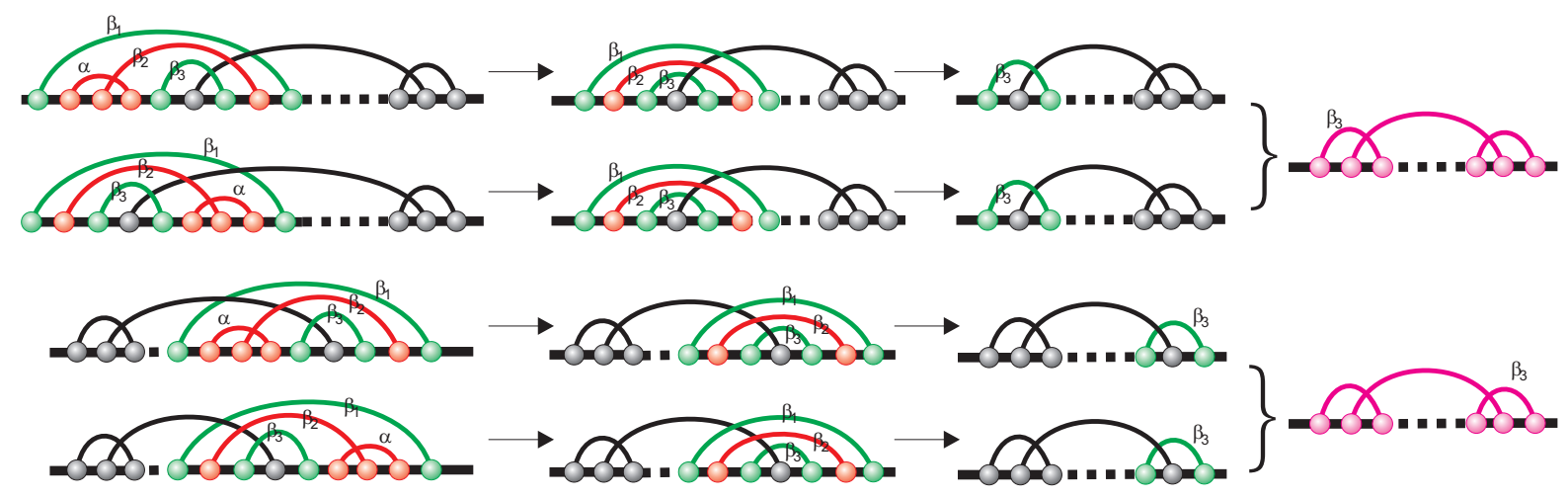

Fig. 35: The term $4 u_{4} i_{k}\left(s-2, u_{1}, u_{2}, u_{3}, u_{4}\right)$.

\subsection{IV.ii}
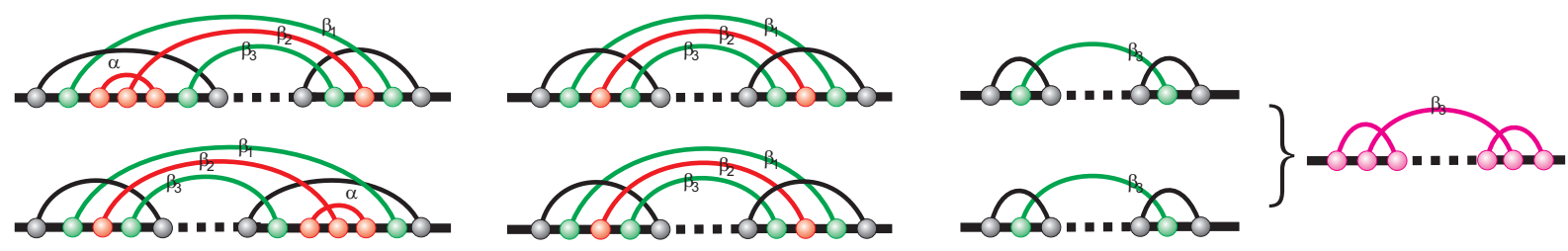

Fig. 36: The term $2\left(u_{4}+1\right) i_{k}\left(s-2, u_{1}, u_{2}, u_{3}, u_{4}+1\right)$.

$$
\left|\mathcal{L}_{2,3}^{\mathbf{C}_{4}}\right|=4 u_{4} i_{k}\left(s-2, u_{1}, u_{2}, u_{3}, u_{4}\right)+2\left(u_{4}+1\right) i_{k}\left(s-2, u_{1}, u_{2}, u_{3}, u_{4}+1\right),
$$

see Fig. 35 and Fig. 36.

It remains to observe that $\beta_{3}$ becomes otherwise a labeled arc in a $\mathcal{J}_{k}\left(s-1, u_{1}, u_{2}, u_{3}, u_{4}\right)$ shape, which is not contained in any $\mathbf{C}_{i}$-element. Thus

$$
\left.\left|\mathcal{L}_{2,3}^{0}\right|=2\left((s-2)-u_{1}-2 u_{2}-2 u_{3}-3 u_{4}\right)\right) i_{k}\left(s-2, u_{1}, u_{2}, u_{3}, u_{4}\right)
$$

and Claim 2.3 follows.

Eq. (4.13) now follows from Claim 1, 2.1, 2.2, and Claim 2.3.

Next we prove eq. (4.14). We choose some $\eta \in \mathcal{J}_{k}\left(s+1, u_{1}, u_{2}, u_{3}, u_{4}+1\right)$ and label one $\mathbf{C}_{4}$-element denoting one of its two 2 -arcs by $\alpha$. We denote the set of these labeled shapes, $\lambda$, by $\mathcal{L}_{*}$. Clearly,

$$
\left|\mathcal{L}_{*}\right|=2\left(u_{4}+1\right) i_{k}\left(s+1, u_{1}, u_{2}, u_{3}, u_{4}+1\right) .
$$

Let $\gamma$ be the arc crossing $\alpha$. The removal of $\alpha$ can lead to either an additional $\mathbf{C}_{2^{-}}$or an additional $\mathbf{C}_{3}$-element in a shape, whence

$$
\mathcal{L}_{*}=\mathcal{L}_{*}^{\mathbf{C}_{2}} \dot{\cup} \mathcal{L}_{*}^{\mathbf{C}_{3}}
$$

where $\mathcal{L}_{*}^{\mathbf{C}_{i}}$ denotes the set of labeled shapes, $\lambda \in \mathcal{L}_{*}$, that induce shapes having a labeled $\mathbf{C}_{i}$-element containing $\gamma$. 
First,

$$
\left|\mathcal{L}_{*}^{\mathbf{C}_{2}}\right|=2\left(u_{2}+1\right) i_{k}\left(s, u_{1}, u_{2}+1, u_{3}, u_{4}\right),
$$

follows by inspection of Fig. 37.

We next observe

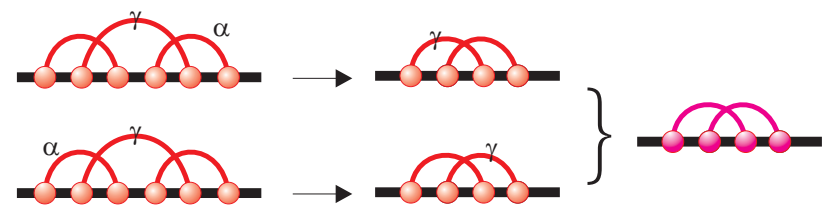

Fig. 37: The term $2\left(u_{2}+1\right) i_{k}\left(s, u_{1}, u_{2}+1, u_{3}, u_{4}\right)$.

$$
\left|\mathcal{L}_{*}^{\mathbf{C}_{3}}\right|=\left(u_{3}+1\right) i_{k}\left(s, u_{1}, u_{2}, u_{3}+1, u_{4}\right) .
$$

see Fig. 38, from which eq. 4.14 immediately follows.

It remains to show that the numbers $i_{k}\left(s, u_{1}, u_{2}, u_{3}, u_{4}\right)$ can be uniquely derived from

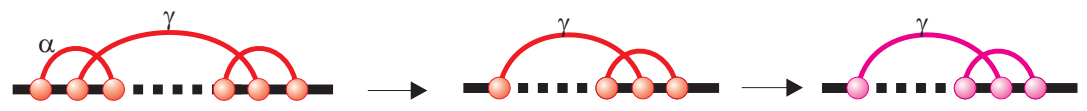

Fig. 38: The term $\left(u_{3}+1\right) i_{k}\left(s, u_{1}, u_{2}, u_{3}+1, u_{4}\right)$.

eq. (4.11), eq. (4.12), eq. (4.13) and eq. (4.14). This follows by induction on $s$.

Acknowledgments. This work was supported by the 973 Project, the PCSIRT Project of the Ministry of Education, the Ministry of Science and Technology, and the National Science Foundation of China.

\section{References}

[1] Chen, W.Y.C., Deng, E.Y.P., Du, R.R.X., Stanley, R.P., Yan, C.H. 2007. Crossings and nestings of matchings and partitions, Trans. Amer. Math. Soc. 359, 1555-1575.

[2] Flajolet, P. and Sedgewick, R. 2009. Analytic combinatorics, Cambridge University Press, New York.

[3] Grabiner, D.J. and Magyar, P. 1993. Random walks in Weyl chambers and the decomposition of tensor powers, J. Algebr. Comb. 2, 239-260.

[4] Han, H. S. W. and Reidys, C. M. 2008. Pseudoknot RNA structures with arc-lenght ? 4. J. Comp. bio., 9, 1195-1208.

[5] Haslinger, C. and Stadler, P.F., 1999. RNA structures with pseudo-knots. Bull. Math. Biol. 61, 437-467.

[6] Henrici, P. 1974. Applied and Computational Complex Analysis volumn 2, John Wiley.

[7] Hofacker, I.L., Schuster, P. and Stadler, P.F. 1998. Combinatorics of RNA secondary structures., Discr. Appl. Math. 88, 207-237. 
[8] Howell, J.A., Smith, T.F., and Waterman, M.S. 1980. Computation of generating functions for biological molecules, SIAM J. Appl. Math. 39, 119-133.

[9] Jin, E.Y., Qin, J. and Reidys, C.M. 2008. Combinatorics of RNA structures with pseudoknots, Bull. Math. Biol. 70, 45-67.

[10] Jin, E.Y. and Reidys, C.M. 2008. Asymptotic enumeration of RNA structures with pseudoknots, Bull. Math. Biol. 70, 951-970.

[11] Jin, E.Y. and Reidys, C.M. 2009. Combinatorial design of pseudoknot RNA, Adv. Appl. Math. 42, 135-151.

[12] Jin, E.Y., Reidys, C.M. and Wang, R.R. 2008. Asympotic analysis of $k$-noncrossing matchings, arXiv:0803.0848.

[13] Ma, G. and Reidys, C.M. 2008. Canonical RNA pseudoknot structures, J. Comput. Biol. 15, 1257-1273.

[14] Penner, R.C. and Waterman, M.S. 1993. Spaces of RNA secondary structures Adv. Math. 101, 31-49.

[15] Reidys, C.M. and Wang, R.R. 2009. Shapes of RNA pseudoknot structures, arXiv:0906.3999.

[16] Reidys, C.M. and Stadler, P.F. 2002. Combinatorial Landscapes, SIAM Reviews, 44, $3-54$.

[17] Rietveld, K., Van Poelgeest, R., Pleij, C.W., Van Boom, J.H., and Bosch, L. 1982. The tRNA-like structure at the $3^{\prime}$ terminus of turnip yellow mosaic virus RNA. Differences and similarities with canonical tRNA. Nucleic Acids Res, 10,1929-1946.

[18] Stanley, R. 1980. Differentiably finite power series, Europ. J. Combinatorics 1, 175188.

[19] Titchmarsh, E.C. 1939.The theory of functions, Oxford Uninversity Press, Oxford, UK.

[20] Wasow, W. 1987. Asymptotic expansions for ordinary differential equations, Dover, New York.

[21] Waterman, M.S. and Smith, T.F. 1978. RNA Secondary Structure: A Complete Mathematical Analysis, Mathematical Bioscience, 42, 257-266.

[22] Waterman, M.S. 1979. Combinatorics of RNA hairpins and cloverleafs, Stud. Appl. Math. 60, 91-96.

[23] Waterman, M.S. and Schmitt, W.R. 1994. Linear trees and RNA secondary structure, Discr. Appl. Math. 51, 317-323. 